\title{
Generic Misalignment Aberration Patterns in Wide-Field Telescopes
}

\author{
Paul L. Schechter \\ MIT Kavli Institute, Cambridge, MA 02139 \\ schech@mit.edu \\ and \\ Rebecca Sobel Levinson \\ MIT Kavli Institute, Cambridge, MA 02139 \\ rsobel@mit.edu
}

\begin{abstract}
Axially symmetric telescopes produce well known "Seidel" off-axis third-order aberration patterns: coma, astigmatism, curvature of field and distortion. When axial symmetry is broken by the small misalignments of optical elements, additional third-order aberration patterns arise: one each for coma, astigmatism and curvature of field and two for distortion. Each of these misalignment patterns is characterized by an associated two-dimensional vector, each of which in turn is a linear combination of the tilt and decenter vectors of the individual optical elements. For an $N$-mirror telescope, $2(N-1)$ patterns must be measured to keep the telescope aligned. Alignment of the focal plane may require two additional patterns. For $N=3$, as in a three mirror anastigmat, there is a two-dimensional "subspace of benign misalignment" over which the misalignment patterns for third-order coma, astigmatism and curvature of field are identically zero. One would need to measure at least one of the two distortion patterns to keep the telescope aligned. Alternatively, one might measure one of the fifth-order misalignment patterns, which are derived herein. But the fifth-order patterns are rather insensitive to misalignments, even with moderately wide fields, rendering them of relatively little use in telescope alignment. Another alternative would be to use telescope pointing as part of the alignment solution.
\end{abstract}

Subject headings: telescopes - optics 


\section{Introduction}

\section{1. new telescopes, stringent constraints}

The designs for several large telescopes that may get built in the next decade are driven largely by the need for superb and stable image quality over wide fields (Ma et al. 2008; Phillion et al. 2006). Perhaps the most demanding of the scientific programs that in turn drive these requirements is that of cosmological weak lensing, using galaxy images to measure gravitational shape distortions as small as as one part in ten thousand.

Of particular concern for ground-based telescopes are the rigid body motions of the

optical elements due to gravitational and thermal stresses on the telescope structure 1 The positions of the optical elements can be controlled only to the extent that they can be measured, putting a premium on the accurate characterization of the aberrations generated by telescope misalignments.

On the assumption that at least some of these aberrations are best measured with wavefront sensors, several questions immediately arise. How many aberrations must be measured? Which ones? At how many field positions must the measurements be made? Where?

One can always, as a last resort, attempt to answer these questions by simulation. But we argue here that the answers to these questions hinge on the identification of generic field aberration patterns that emerge in a wide variety of circumstances. A relatively small number of such patterns suffices to efficiently diagnose and correct misalignments. And only a small number of field points must be sampled to measure these patterns. Moreover two of the patterns may in some cases be determined directly from science data.

\section{2. literature}

The practical astronomical literature on the alignment of wide field telescopes is very limited. McLeod's (1996) paper describing the use of coma and astigmatism patterns to align the Whipple Observatory 1.2-m telescope anchors the recent literature. Wilson \& Delabre (1997) discuss the alignment of the ESO NTT. Gitton \& Noethe (1998) describe the alignment of the ESO VLTs, and Noethe \& Guisard (2000) give a more general description of the astigmatism patterns expected from two-mirror telescopes. Lee et al. (2008) give a general

\footnotetext{
${ }^{1}$ Such telescopes are also subject to deformations of the optical elements, but on longer timescales than the rigid body motions.
} 
treatment of third-order misalignment distortions and then discuss coma and astigmatism and curvature of field in their case studies. Palunas et al. (2010) describe the alignment of the Magellan Nasmyth telescopes using coma, astigmatism and curvature of field.

Maréchal (1950) derives the third-order misalignment aberration patterns for coma, astigmatism, curvature of field and distortion.

Thompson and collaborators (Shack \& Thompson 1980; Thompson 2005; Thompson et al 2009) develop a formalism for analyzing telescope misalignments using a vector notation that is elegant and relatively transparent. It isolates generic misalignment patterns associated with third-order aberrations - coma, astigmatism, curvature of field and distortion - and beyond that, generic misalignment patterns associated with fifth-order aberrations.

In an unpublished M.S. thesis, Tessieres (2003) used ray tracing software to determine amplitudes for Thompson's misalignment patterns, which at that time had only appeared in Thompson's Ph.D. thesis (1980). Hvisc \& Burge (2008) build on Tessieres' work in modeling a four mirror corrector for the Hobby-Ebberly Telescope. They identify the linear combinations of orthogonal aberration patterns (integrated over the field) that are most sensitive to the tilts and decenters of the mirrors.

\section{3. outline}

In the following sections we rederive these same misalignment aberration patterns, using the Thompson et al. vector notation but following instead the development of Schroeder (1987). The present paper is quite similar in spirit to Tessieres': identify those aberration patterns of potential interest and ascertain which are of greatest value in aligning a telescope. However our approach differs in that, in the interest of efficiency, we ignore patterns that are non-linear in the tilts and decenters of the mirrors. This simplification is appropriate for small misalignments of an otherwise rotationally symmetric telescope. We also give greater consideration to the role of distortion, curvature of field and spherical aberration than did Tessieres.

In $\oint_{2}$ we discuss the misalignment patterns produced by two-mirror telescopes, proceeding from the better known generic coma and astigmatism misalignment patterns, through the almost trivial curvature of field misalignment pattern, to the two distortion misalignment patterns. We then retrace our steps using a more general approach that shows how the misalignment patterns produced by an optic derive from the surface of that optic. In \$3 we discuss the alignment of three-mirror telescopes. 
In $\$ 4$ we use the same methods used in \$2 to deduce the misalignment patterns associated with fifth-order aberrations. In the course of this we attempt to systematize the somewhat ragged nomenclature associated with the fifth-order aberrations. We additionally examine the relative magnitudes of the fifth-order aberrations, which cast some doubt on their practical utility for telescope alignment. In \$5 we discuss the number and placement of wavefront sensors needed to align a three mirror telescope. In 96 we address a variety of complicating factors: mirror deformations, transmitting correctors, central obscurations and focal plane tilts. In 97 we discuss several ways in which the misalignment aberration patterns might be used, and why one might ultimately choose to forego their use.

\section{Generic patterns and two-mirror telescopes}

\section{1. coma}

McLeod's (1996) paper shows how an astigmatic misalignment pattern can be used in conjunction with coma to align a telescope. McLeod's first step is to center the secondary so as to zero the coma. He does not explicitly refer to a coma "pattern," but it is widely appreciated that decentering the secondary of a Ritchey-Chretien telescope produces coma that is to first order constant across the field. For the present purposes we take this to be a pattern, albeit a boring one. McLeod does identify an astigmatism pattern, which he then renders symmetric by rotating the secondary about its coma-free pivot.

Schroeder (1987) calculates the coma patterns that arise in a two mirror telescope, allowing for tilting and decentering the secondary. The comatic wavefront $G^{\text {coma }}$ is a function of position on the pupil $\vec{\rho}$, with polar coordinates $\rho$ and $\phi$, and field angle $\vec{\sigma}$, with polar coordinates $\sigma$ and $\theta$ :

$$
G^{\text {coma }}=G_{\text {Seidel }}^{c o m a} \sigma \rho^{3} \cos (\phi-\theta)+G_{\text {decenter }}^{c o m a} \rho^{3} \cos \left(\phi-\phi_{\ell}\right)+G_{\text {tilt }}^{c o m a} \alpha \rho^{3} \cos \left(\phi-\phi_{\alpha}\right)
$$

where $\vec{\ell}$ is the decenter of the secondary which projects to angle $\phi_{\ell}$ on the pupil, and $\vec{\alpha}$ is the vector tilt of the secondary which projects to angle $\phi_{\alpha}$ on the pupil. The first term on the right hand side gives the symmetric "Seidel" coma typical of an aligned two mirror telescope. The next two terms give the constant coma pattern typical of a decentering or tilt of the secondary. The coefficients $G_{\text {Seidel }}^{\text {coma }}, G_{\text {decenter }}^{\text {coma }}$, and $G_{\text {tilt }}^{\text {coma }}$ depend upon the radii of curvature, $R_{i}$, and conic constants, $K_{i}$, of the two mirrors, the indices of refraction of the material (air) preceding the mirrors, $n_{i}$, the positions, $s_{i}$, and magnifications, $m_{i}$ of the object for each mirror, and the distance from the primary mirror to the secondary, $W$. In a 
Ritchey-Chretien telescope the $G_{\text {Seidel }}^{c o m a}$ term is identically zero, giving no coma when aligned. The notation here is different from that of Schroeder; the conversion from Schroeder's to the present notation is given in appendix A.

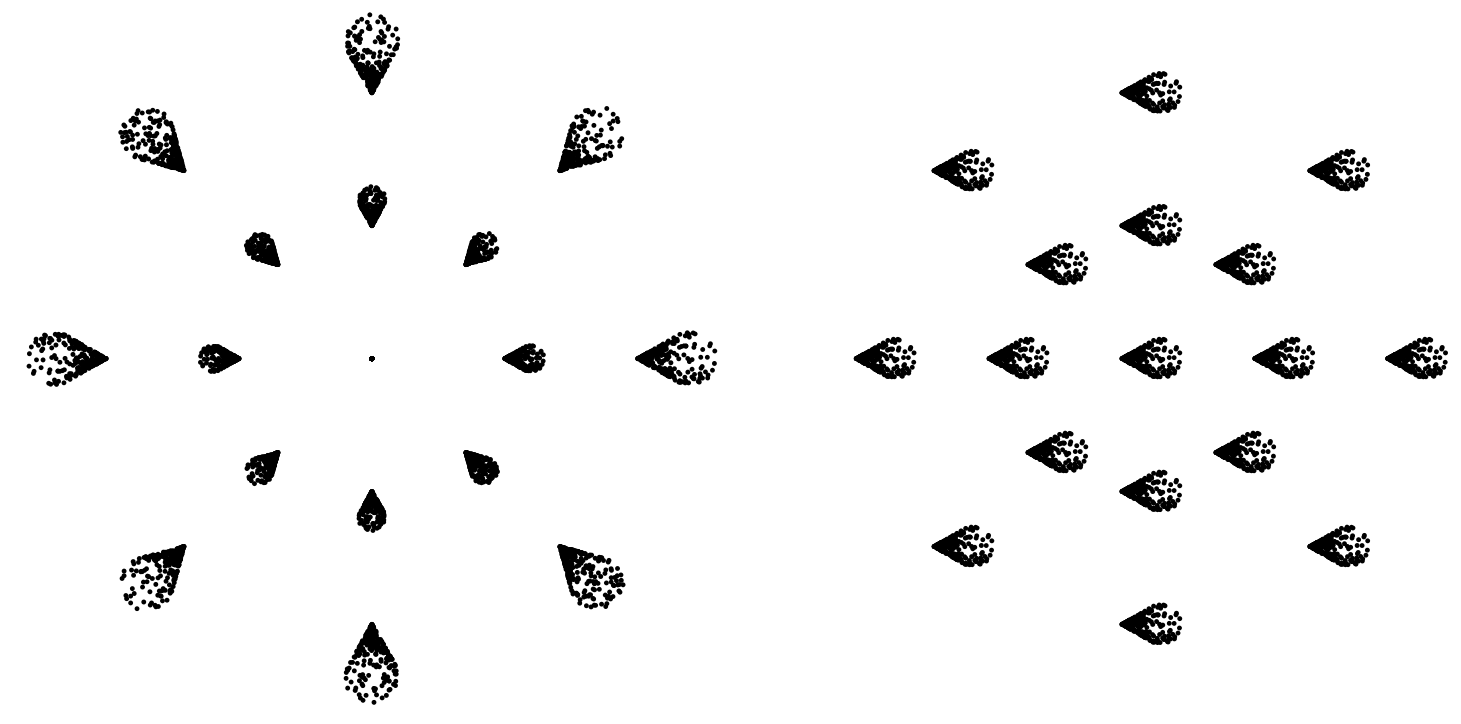

Fig. 1.- Comatic field patterns. (a) Seidel coma field pattern typical of an aligned telescope. (b) Constant coma indicating a tilt and (or) decentering of the secondary along the $\mathrm{x}$ axis.

The Seidel term varies linearly as field angle $\sigma$ and as $\rho^{3} \cos \phi$ and (or) $\rho^{3} \sin \phi$ on the pupil. The tilt and decenter terms are constant over the field, but have the same functional dependence on pupil coordinates as the first term. We shall somewhat loosely refer to any aberration that has the same dependence upon pupil coordinates as "coma," even when it does not have the Seidel coma dependence on field angle $\sigma$. Figure 1a shows the point spread function at various points in the field for the first term in equation (1). Figure 1b shows the point spread function pattern typical of either of the last two terms in equation (1).

In practice, the constant coma pattern shown in Figure 1 $\mathrm{b}$ will be superimposed on the Seidel pattern if one is present. We here plot the two patterns separately as the patterns have different physical motivations and thus provide different information about the telescope. The Seidel pattern is a result of the telescope design, and fitting for that pattern provides no information about tilts or decenterings of the mirrors. In contrast, the magnitude and orientation of the constant coma pattern will provide information about a telescope's alignment. 


\section{2. astigmatism}

Following Schroeder's (1987) example for coma, McLeod (1996) calculated the corresponding astigmatism pattern for the case of a nulled field constant coma pattern. He finds an astigmatic wavefront, $G^{\text {astig }}$, given by:

$$
G^{\text {astig }}=G_{\text {sym }}^{\text {astig }} \sigma^{2} \rho^{2} \cos 2(\phi-\theta)+G_{\text {decenter }}^{\text {astig }} \sigma \ell \rho^{2} \cos \left(2 \phi-\theta-\phi_{\ell}\right)+G_{\text {tilt }}^{\text {astig }} \sigma \alpha \rho^{2} \cos \left(2 \phi-\theta-\phi_{\alpha}\right)
$$

The first term on the right gives the symmetric astigmatism typical of an aligned two mirror telescope. The next two terms give the astigmatism pattern typical of a decentering or tilt of the secondary. The coefficients $G_{s y m}^{a s t i g}, G_{d e c e n t e r}^{a s t i}$ and $G_{t i l t}^{a s t i g}$ again depend upon the radii of curvature, $R_{i}$ and conic constants, $K_{i}$ of the two mirrors, the indices of refraction of the air preceding the mirrors, $n_{i}$, the positions, $s_{i}$ and magnifications, $m_{i}$ of the object for each mirror, and the distance from the primary mirror to the secondary, $W$. The details of the conversion from McLeod's notation to the above are given in appendix B.
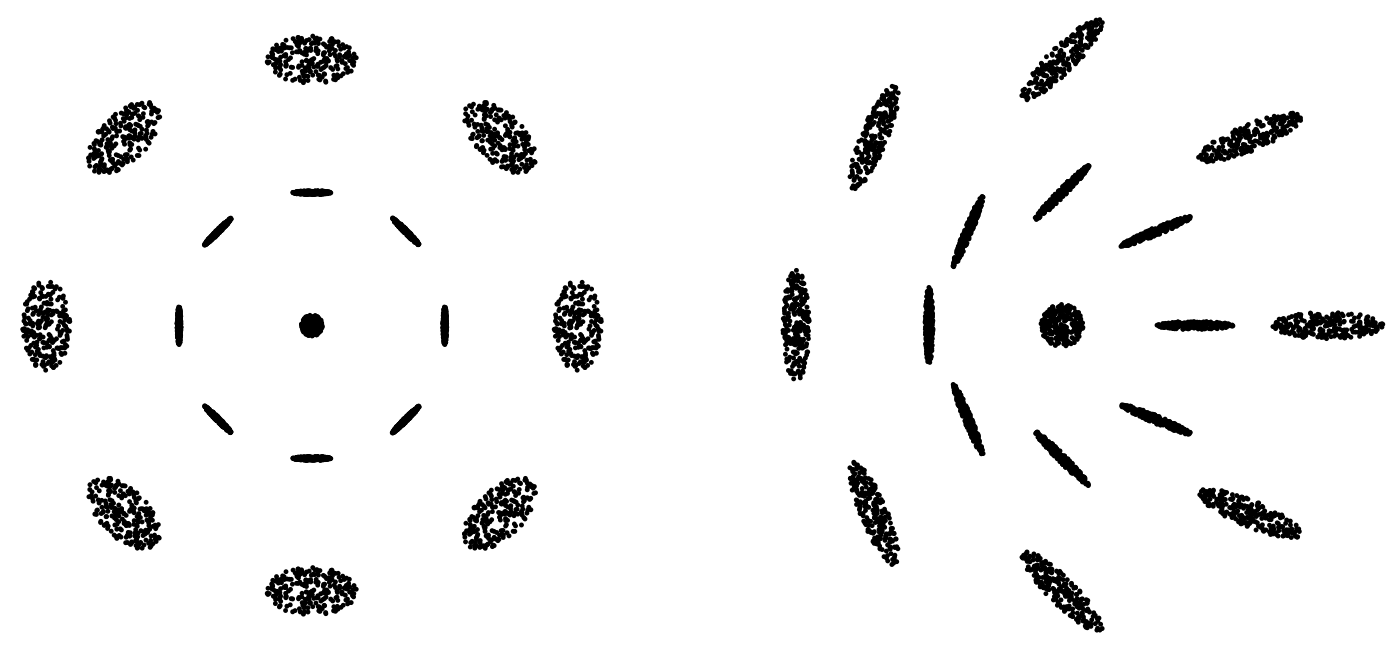

Fig. 2.- Astigmatic field patterns. (a) Seidel astigmatism field pattern typical of an aligned telescope. A constant defocus has been added to show the orientation of the astigmatism. (b) Astigmatic field pattern indicating a tilt and (or) decentering of the secondary along the $\mathrm{x}$ axis.

The symmetric term varies as the square of the field radius $\sigma$ and varies as $\rho^{2} \cos 2 \phi$ and (or) $\rho^{2} \sin 2 \phi$ on the pupil. This is almost, but not quite the variation associated with 
third-order, Seidel astigmatism.2 It is readily decomposed into terms that vary as $\rho^{2} \cos ^{2} \phi$ (Seidel astigmatism) and $\rho^{2} \cos ^{0} \phi$ (Seidel curvature of field). The tilt and decenter terms have the same functional dependence on pupil coordinates as the symmetric term, but vary linearly with distance from the center of the field $\sigma$, and vary as the cosine and (or) sine of the field angle $\theta$. We shall again refer loosely to any aberration that has the same dependence upon pupil coordinates as astigmatism, even when it does not have the Seidel dependence on field position. Figure 2 a shows the point spread function at various points in the field for the symmetric term in equation (2). Figure $2 \mathrm{~b}$ shows the point spread function pattern characteristic of either the tilt or decenter terms in equation (2) 3

As with the comatic aberrations, the astigmatic field pattern shown in Figure $2 \mathrm{~b}$ will in general be superimposed on the Seidel pattern if one is present. Some previous treatments have referred to this superposition of field patterns as a single misalignment pattern; McLeod (1996) describes a single astigmatism pattern, which he symmetrizes in the course of aligning his telescope; Thompson (2005) refers to a binodal astigmatism pattern which results from misalignments. In this paper we decompose the astigmatism into two patterns, a symmetric one characteristic of an aligned telescope and an asymmetric one introduced by misalignments. The asymmetric pattern described by McLeod (1996) and the binodal pattern plotted by Thompson (2005) are produced by superimposing the symmetric and asymmetric patterns in Figures $2 \mathrm{a}$ and $2 \mathrm{~b}$. The nodes are simply field positions where the symmetric Seidel and asymmetric misalignment patterns have cancelled 4

Both for coma and for astigmatism (and in the cases of the additional aberrations considered below) the misalignment pattern varies as one power of field radius less rapidly than the corresponding symmetric pattern.

\footnotetext{
${ }^{2}$ By convention "Seidel" astigmatism is taken to vary as $\rho^{2} \cos ^{2} \phi$ on the pupil. By contrast "Zernike" astigmatism is almost always taken to vary as $\rho^{2} \cos 2 \phi$. The Seidel definition emerges naturally from the derivation of aberration patterns. The Zernike definition makes for more symmetric wavefronts and orthogonality among the different aberrations. A similar ambiguity arises in the definition of trefoil.

${ }^{3}$ The "dreamcatcher" plot of Figure $2 \mathrm{~b}$ makes cameo appearances in a number of contexts. It can be seen in a map of image elongations at the prime focus of the LBT (Romano et al. 2010) and in the point spread function map, Figure 4.14, in version 12.0 of the Chandra Proposer's Observatory Guide (Chandra X-rav Center 2009). The first such plot of which the authors are aware is in the paper by Shack \& Thompson (1980). Maréchal (1950) comes close plotting the magnitude and orientation of the misalignment astigmatism pattern but suppressing the sign.

${ }^{4}$ Terms that are nonlinear in the misalignments also contribute to the positions of the nodes. Most interestingly, the inclusion of a non-linear term may rotate the orientation of the nodes by $90^{\circ}$. However, the inclusion of terms that are nonlinear in the misalignment has no effect on the underlying Seidel or linear patterns.
} 


\section{3. curvature of field}

Coma and astigmatism are just two of the five third-order Seidel aberrations. Curvature of field (henceforth COF) manifests itself as a defocus that varies as the square of the distance from the center of an assumed flat focal plane. McLeod might in principal have used curvature of field (rather than astigmatism) to align the Whipple 1.2-m, but there is a potential degeneracy with a tilted instrument. Following Schroeder and McLeod, we find an associated wavefront,

$$
G^{C O F}=G_{\text {Seidel }}^{C O F} \sigma^{2} \rho^{2}+G_{\text {decenter }}^{C O F} \sigma \ell \rho^{2} \cos \left(\theta-\phi_{\ell}\right)+G_{\text {tilt }}^{C O F} \sigma \alpha \rho^{2} \cos \left(\theta-\phi_{\alpha}\right)
$$

The first term on the right hand side gives the symmetric Seidel curvature of field typical of an aligned two mirror telescope, and the next two give the defocus patterns typical of a decenter or tilt of the secondary. The coefficients $G_{\text {Seidel }}^{C O F}, G_{\text {decenter }}^{C O F}$, and $G_{\text {tilt }}^{C O F}$ again depend upon the radii of curvature, $R_{i}$ and conic constants, $K_{i}$ of the two mirrors, the indices of refraction of the air preceding the mirrors, $n_{i}$, the positions, $s_{i}$ and magnifications, $m_{i}$ of the object for each mirror, and the distance from the primary mirror to the secondary, $W$. The details of the derivation of the above are given in appendix $\mathrm{C}$.

The Seidel term varies as the square of the field radius $\sigma$ and varies as $\rho^{2}$ on the pupil. But the dependence on pupil position is exactly the same as that of defocus, which is a first-order aberration. Anticipating the nomenclature introduced in \$4 below, curvature of field might equally well be called "third-order defocus," but we bow to convention. The tilt and decenter terms vary linearly with distance from a line passing through the center of the field, but have the same functional dependence on pupil coordinates. This is precisely what one would expect for a tilted focal plane. Figure 3 a shows the point spread function at various points in the field for the Seidel term in equation (3). Figure $3 \mathrm{~b}$ shows the point spread function pattern introduced by either the tilt or decenter terms in equation (3).

\section{4. distortion}

While distortion is one of the five Seidel aberrations, for many purposes it can be neglected, since it does not degrade image quality. Distortion does, however alter the positions of images in the field, and of particular interest for the measurements of weak gravitational lensing, it changes the shapes of extended objects. And most importantly for the present discussion it may be of some use in aligning a telescope.

Following the conventions of Schroeder and McLeod, we find the associated wavefront 


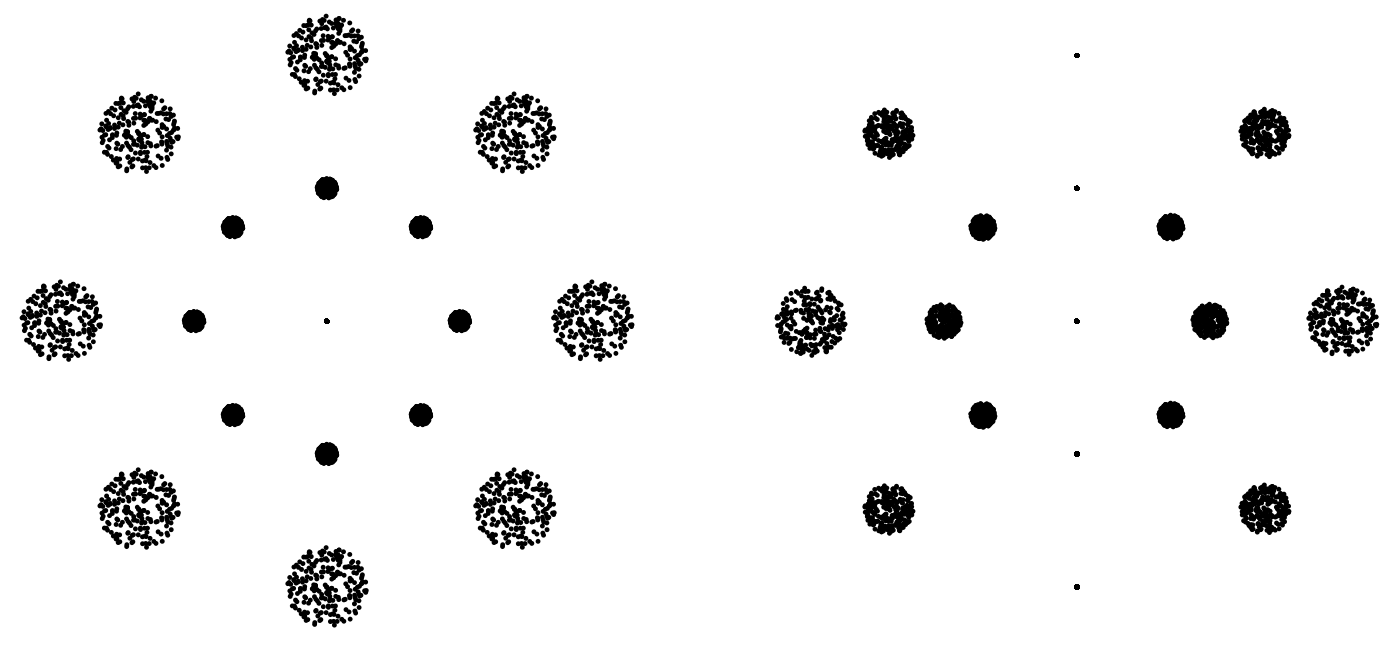

Fig. 3.- Curvature of field patterns. (a) Seidel COF typical of an aligned telescope. (b) COF field indicating a tilt and (or) decenter of the secondary along the $\mathrm{x}$ axis.

delay for a misaligned two mirror telescope:

$$
\begin{aligned}
G^{\text {distortion }} & =G_{\text {Seidel }}^{\text {distortion }} \sigma^{3} \rho \cos (\phi-\theta) \\
& +G_{\text {decenter }, \sigma}^{\text {distortion }} \sigma^{2} \ell \cos \left(\theta-\phi_{\ell}\right) \rho \cos (\phi-\theta)+G_{\text {decenter }, \rho}^{\text {distortion }} \sigma^{2} \ell \rho \cos \left(\phi-\phi_{\ell}\right) \\
& +G_{\text {tilt }, \sigma}^{\text {distortion }} \sigma^{2} \alpha \cos \left(\theta-\phi_{\alpha}\right) \rho \cos (\phi-\theta)+G_{\text {tilt }, \rho}^{\text {distion }} \sigma^{2} \alpha \rho \cos \left(\phi-\phi_{\alpha}\right)
\end{aligned}
$$

The Seidel term on the right varies as the cube of field angle $\sigma$ and as $\rho \cos \phi$ and (or) $\rho \sin \phi$ on the pupil. Distortion differs from coma, astigmatism and curvature of field in having two distinct misalignment aberration patterns rather than only one. We will encounter several similar pairs of misalignment aberration patterns when we consider fifth-order misalignment patterns in $\S 4$. The two terms are distinguished by whether the direction of the tilt, $\vec{\alpha}$ or decenter, $\vec{\ell}$ enters in a dot product with the field position, $\vec{\sigma}$ or the pupil position, $\vec{\rho}$. We use " $\sigma$ " and " $\rho$ " to label the two alternatives.

The $\sigma$ terms have the same functional dependence on pupil coordinates as the Seidel term, but are proportional to the square of the field angle $\sigma$ and the cosine of its polar coordinate, $\theta$. These produce a field distortion pattern directed radially outward, but with a magnitude that depends on the product of the field angle and its projection onto the decenter or tilt.

The $\rho$ terms also have the same functional dependence on pupil coordinates as the first, 
but vary only as the square field angle $\sigma$. The direction of the distortion is that of the decenter or tilt, but its magnitude increases outward as the square of the distance from the center of the field.

The three distortion patterns are shown in figure 4. Details of the derivation of the coefficients are given in appendix D.

Each of the two distinct misalignment distortion patterns is characterized by a twovector. Were one able to measure those vectors with the same accuracy as the two-vectors that characterize coma and astigmatism one might in principle use only distortion measurements to align a two mirror telescope.

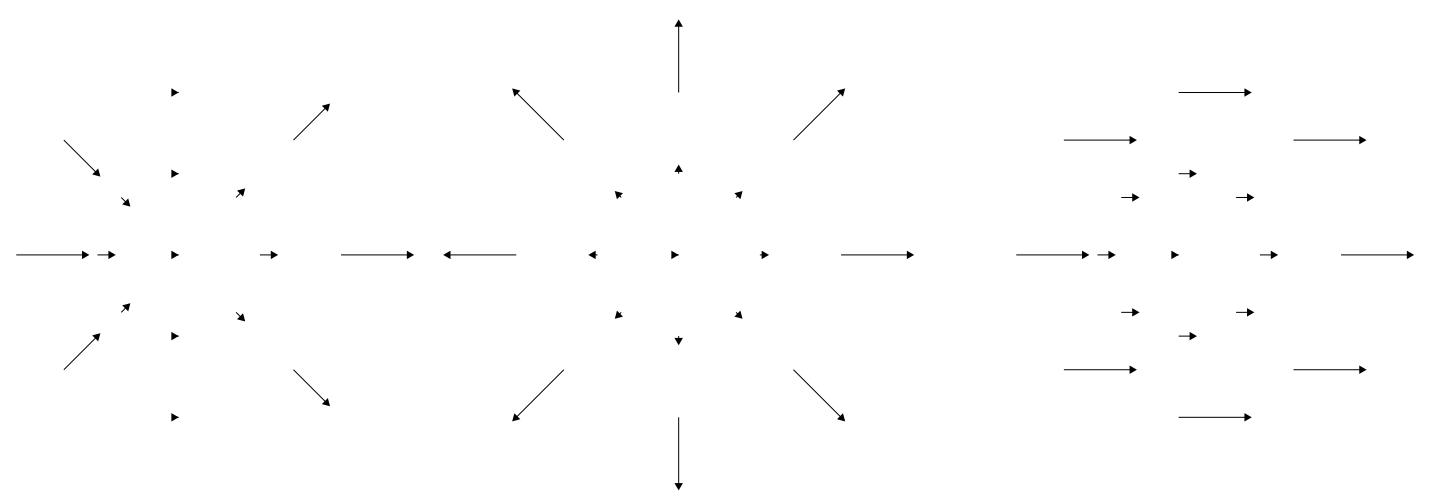

Fig. 4.- Distortion field patterns. (a) The " $\sigma$ " distortion pattern indicating a tilt and (or) decentering of the secondary along the $\mathrm{x}$ axis. (b) Seidel distortion typical of an aligned telescope. (c) The " $\rho$ " distortion pattern indicating a tilt and (or) decentering of the secondary along the $\mathrm{x}$ axis.

\section{5. spherical aberration}

Spherical aberration is the fifth of the Seidel aberrations and is constant across the field. Tilts and decenters do not produce asymmetric spherical aberration patterns.

\section{6. generalization}

In the preceding subsections the third-order aberrations were cast so as to display explicitly the azimuthal dependence upon pupil position, $\vec{\rho}$ and field angle, $\vec{\sigma}$. These can be 
recast more compactly and transparently in vector form. For example the Seidel distortion term above varies as $(\vec{\sigma} \cdot \vec{\sigma})(\vec{\sigma} \cdot \vec{\rho})$.

Suppose a single mirror $i$ serves as its own pupil. The wavefront delay $G^{3 r d}$ for a ray that intercepts the mirror at position $\vec{\varpi}$ and that makes an angle $\vec{\psi}$ with the axis of the mirror is given by 5

$$
\begin{aligned}
G^{3 r d} & =W_{040}\left(\frac{\vec{\varpi}}{R} \cdot \frac{\vec{\varpi}}{R}\right)\left(\frac{\vec{\varpi}}{R} \cdot \frac{\vec{\varpi}}{R}\right)+W_{131}\left(\vec{\psi} \cdot \frac{\vec{\varpi}}{R}\right)\left(\frac{\vec{\varpi}}{R} \cdot \frac{\vec{\varpi}}{R}\right) \\
& +W_{222}\left(\vec{\psi} \cdot \frac{\vec{\varpi}}{R}\right)\left(\vec{\psi} \cdot \frac{\vec{\varpi}}{R}\right)+W_{220}(\vec{\psi} \cdot \vec{\psi})\left(\frac{\vec{\varpi}}{R} \cdot \frac{\vec{\varpi}}{R}\right) \\
& +W_{311}(\vec{\psi} \cdot \vec{\psi})\left(\vec{\psi} \cdot \frac{\vec{\varpi}}{R}\right)
\end{aligned}
$$

where $W_{040}$ is the spherical aberration coefficient, $W_{131}$ is the coma coefficient, $W_{222}$ is the astigmatism coefficient, $W_{220}$ is the curvature of field coefficient, and $W_{311}$ is the distortion coefficient. These aberration coefficients depend only on the curvature of the mirror, $R$, the conic constant of the mirror, $K$, the magnification of the mirror, $m$, the position of the object for the mirror, $s$, and the index of refraction immediately preceding the mirror, $n 6$. In $\S 5.1$ of his book Schroeder (1987) shows that the aberrations which vary linearly with ray height on the optic must be zero for conic section optics which serve as their own pupils. We therefore set $W_{311}$ to zero and ignore it in the following discussion. The values for the other coefficients are given in Table 1.

Following Schroeder (1987), one finds that if the pupil is offset by $W$ along the axis of

Table 1. Aberration coefficient values for the optic-centered pupil.

\begin{tabular}{cccc}
\hline \hline$W_{040}$ & $W_{131}$ & $W_{222}$ & $W_{220}$ \\
\hline$\frac{n R}{4}\left(\left(\frac{R}{s}-1\right)^{2}+K\right)$ & $-n R\left(\frac{R}{s}-1\right)$ & $n R$ & 0 \\
\hline
\end{tabular}

\footnotetext{
${ }^{5}$ We reserve $\vec{\rho}$ for the position on the pupil when the optic is despaced from the pupil.

${ }^{6}$ We adopt the convention of Schroeder (1987) whereby rays traveling in opposite directions encounter oppositely signed indices of refraction; a ray incident on the primary mirror through air $(n=1)$ will encounter a negative index of refraction $(n=-1)$ once reflected and traveling towards the secondary. Therefore, although this discussion deals primarily with mirrors and thin lenses, the index of the refraction of the material surrounding the optics cannot be entirely ignored.
} 
the mirror, the position at which a ray lands on the mirror, $\vec{\varpi}$ depends upon its position on the pupil, $\vec{\rho}$ and the angle the chief ray makes to the pupil normal, $\vec{\sigma}$ (which is defined to be the field angle), and upon $W$. The angle that the ray makes with the axis of the mirror, $\vec{\psi}$ depends upon field angle $\vec{\sigma}$ and the pupil offset, $W$.

If the mirror is tilted by angle $\vec{\alpha}$, it changes the angle $\vec{\psi}$ that a ray makes with the mirror's axis. And if the mirror is decentered from the optical axis (which is perpendicular to and centered on the pupil) by $\vec{\ell}$, it changes both the position at which a ray lands on the mirror, $\varpi$ and the angle $\vec{\psi}$ that the ray makes with respect to the mirror's axis.

The net effect of a pupil offset or a misalignment of the mirror relative to the pupil is to shift the position $\vec{\varpi}$ at which a given ray strikes the mirror and to change the angle $\vec{\psi}$ that the ray makes with the axis of the mirror. The transformations from pupil coordinates, $\vec{\rho}$ and field angle, $\vec{\sigma}$ to mirror coordinates and mirror angle for a mirror despaced by an amount $W$ and decentered and tilted by $\vec{l}$ and $\vec{\alpha}$ are then 7

$$
\begin{aligned}
\vec{\psi} & =\left(1-\frac{W}{s}\right) \vec{\sigma}-\left(\vec{\alpha}+\frac{\vec{l}}{s}\right) \\
\vec{\varpi} & =(\vec{\rho}-W \vec{\sigma})-\vec{l} .
\end{aligned}
$$

An offset of the pupil from the mirror by an amount $W$ causes what was Seidel spherical aberration to manifest itself as a combination of spherical aberration, coma, astigmatism, curvature of field and distortion, all of which have the symmetric Seidel field dependence. Likewise what was coma manifests itself as a combination of coma, astigmatism, curvature of field and distortion. This cascade downward from spherical to coma to astigmatism and

\footnotetext{
${ }^{7}$ In deriving equations (6) and (7) we assume that all of the effects of the curvature of an optic on aberrations are embodied in equation (5), and we subsequently treat displacements, $W$, decenters, $\vec{\ell}$ and tilts, $\vec{\alpha}$ of an optic as displacements, decenters, and tilts of the flat surface defined by the plane containing the vertex of that optic. This is equivalent to using a first order approximation of pupil and field coordinates. While this assumption reproduces the stop shift formulae given in Schroeder (1987) and Wilson (1996), it is not strictly correct in the cases of large or very curved mirrors, large fields, or cases where the stop is quite close to the optic. In particular, when a telescope's primary mirror is the aperture stop, the computed aberrations are only correct when $W$ for the primary mirror is set equal to zero, even though the physical aperture is defined by the edge of the optic, which may actually be a finite distance from the optic's vertex. Setting $W$ equal to zero in this special case is natural, as equation (5) completely describes the aberrations introduced by an optic that is its own pupil, without any need for modification. However, this inconsistency would seem to indicate that the assumptions that predicate equations (6) and (7) may need to be modified in order to compute higher order aberrations.
} 
curvature of field and finally to distortion is embodied in the "stop shift" formulae (e.g. Wilson 1996).

Decenterings and tilts of the mirror relative to the pupil also produce cascades. For an axially symmetric telescope we may assume that these tilts and decenters are small and ignore terms that are quadratic and higher in either or both. In the aberration patterns associated with the surviving linear terms, a field angle vector $\vec{\sigma}$ is replaced by either a tilt, $\vec{\alpha}$ or a decenter, $\vec{\ell} / R$. The field angle exponents for the misalignment aberration patterns are therefore smaller by one than those of the corresponding Seidel aberrations.

Table 2 gives aberration patterns that arise when a mirror is offset by $W$ with respect to its pupil, and decentered and tilted by small amounts.

The different signs for odd and even numbered mirrors arise from the fact that the chief ray for a given optic may be traveling in the opposite angular direction than the chief ray on the pupil for the primary mirror, which here determines the field angle $\vec{\sigma}$. Put otherwise, the sign accounts for the fact that preceding mirrors flipped the images. It does not account for the changing indices of refraction which are contained in the $W_{k l m}$ coefficients. The $\sigma$-type distortion patterns have factors $\vec{\sigma} \cdot \frac{\vec{l}}{R}$ or $\vec{\sigma} \cdot \vec{\alpha}$. The $\rho$-type distortion patterns have factors $\vec{\rho} \cdot \frac{\vec{l}}{R}$ or $\vec{\rho} \cdot \vec{\alpha}$.

\section{7. application to 2-mirror telescopes and 2.5-mirror telescopes}

Table 2 of the previous subsection gives the third-order aberrations for a single mirror with a pupil offset by $W$ along the optical axis. The aberrations for a 2-mirror telescope are found by computing the elements of two such tables, one for the primary and one for the secondary, and adding.

Before one can use Table 2 to compute aberrations, one must first know the location of the center of the field as it is defined by the pointing of the pupil. If the center of pointing is unknown then one must add an additional vector variable to the table, $\vec{m}$, which maps the true field coordinate, $\vec{\sigma}$ to that adopted for measurement, $\overrightarrow{\sigma^{\prime}}$ via the relation $\vec{\sigma} \rightarrow \overrightarrow{\sigma^{\prime}}-\vec{m}$. This focal plane decenter will mathematically map Seidel terms to misalignment terms, but it will not physically aberrate the images. For the sake of simplicity, we here assume that the center of pointing is known and treat a decentered focal plane as a 'complication' in \$6.

For many two-mirror telescopes the pupil is coincident with the primary. If one also takes the primary to define the pointing of the telescope, one can take the pupil offset, $W_{1}$,

the mirror decenter, $\vec{\ell}_{1}$ and the mirror tilt, $\vec{\alpha}_{1}$ all to be zero. The misalignment patterns 
Table 2. Symmetric and asymmetric aberration patterns for mirrors offset, decentered and tilted with respect to the pupil.

\begin{tabular}{|c|c|c|c|}
\hline aberration & \multicolumn{2}{|l|}{ pupil offset $W$} & tilt $\vec{\alpha}$ \\
\hline spherical & $\left(\frac{\vec{\rho}}{R} \cdot \frac{\vec{\rho}}{R}\right)\left(\frac{\vec{\rho}}{R} \cdot \frac{\vec{\rho}}{R}\right) \times W_{040}$ & & \\
\hline coma & $\begin{array}{l}\left(\frac{\vec{\rho}}{R} \cdot \frac{\vec{\rho}}{R}\right)\left(\frac{\vec{\rho}}{R} \cdot \vec{\sigma}\right) \times \mp^{\mathrm{a}}[ \\
\quad\left(\frac{W}{s}-1\right) W_{131} \\
\left.\quad+4 \frac{W}{R} W_{040}\right]\end{array}$ & $\begin{array}{l}\left(\frac{\vec{\rho}}{R} \cdot \frac{\vec{\rho}}{R}\right)\left(\frac{\vec{\rho}}{R} \cdot \frac{\vec{l}}{R}\right) \times[ \\
\quad-\left(\frac{R}{s}\right) W_{131} \\
\left.\quad-4 W_{040}\right]\end{array}$ & $\begin{array}{c}\left(\frac{\vec{\rho}}{R} \cdot \frac{\vec{\rho}}{R}\right)\left(\frac{\vec{\rho}}{R} \cdot \vec{\alpha}\right) \times[ \\
-W_{131}\end{array}$ \\
\hline astigmatism & $\begin{aligned}\left(\frac{\vec{\rho}}{R} \cdot \vec{\sigma}\right) & \left(\frac{\vec{\rho}}{R} \cdot \vec{\sigma}\right) \times[ \\
& \left(\frac{W}{s}-1\right)^{2} W_{222} \\
& +2 \frac{W}{R}\left(\frac{W}{s}-1\right) W_{131} \\
& \left.+4 \frac{W^{2}}{R^{2}} W_{040}\right]\end{aligned}$ & $\begin{array}{l}\left(\frac{\vec{\rho}}{R} \cdot \vec{\sigma}\right)\left(\frac{\vec{\rho}}{R} \cdot \frac{\vec{l}}{R}\right) \times \pm^{\mathrm{a}}[ \\
\quad 2\left(\frac{R}{s}\right)\left(\frac{W}{s}-1\right) W_{222} \\
\quad+2\left(\frac{2 W}{s}-1\right) W_{131} \\
\quad+8 \frac{W}{R} W_{040}\end{array}$ & $\begin{array}{l}\left(\frac{\vec{\rho}}{R} \cdot \vec{\sigma}\right)\left(\frac{\vec{\rho}}{R} \cdot \vec{\alpha}\right) \times \pm^{\mathrm{a}}[ \\
\quad 2\left(\frac{W}{s}-1\right) W_{222} \\
\quad+2 \frac{W}{R} W_{131}\end{array}$ \\
\hline $\mathrm{COF}$ & $\begin{aligned} &\left(\frac{\vec{\rho}}{R} \cdot \frac{\vec{\rho}}{R}\right)(\vec{\sigma} \cdot \vec{\sigma}) \times[ \\
&\left(\frac{W}{s}-1\right)^{2} W_{220} \\
&+\frac{W}{R}\left(\frac{W}{s}-1\right) W_{131} \\
&\left.+2 \frac{W^{2}}{R^{2}} W_{040}\right]\end{aligned}$ & $\begin{aligned} &\left(\frac{\vec{\rho}}{R} \cdot \frac{\vec{\rho}}{R}\right)\left(\vec{\sigma} \cdot \frac{\vec{l}}{R}\right) \times \pm^{\mathrm{a}}[ \\
& 2\left(\frac{R}{s}\right)\left(\frac{W}{s}-1\right) W_{220} \\
&+\left(\frac{2 W}{s}-1\right) W_{131} \\
&\left.+4 \frac{W}{R} W_{040}\right]\end{aligned}$ & $\begin{aligned}\left(\frac{\vec{\rho}}{R} \cdot \frac{\vec{\rho}}{R}\right)(\vec{\sigma} \cdot \vec{\alpha}) \times \pm^{\mathrm{a}}[ \\
\quad 2\left(\frac{W}{s}-1\right) W_{220} \\
\quad+\frac{W}{R} W_{131}\end{aligned}$ \\
\hline distortion & $\begin{aligned}\left(\frac{\vec{\rho}}{R} \cdot \vec{\sigma}\right)(\vec{\sigma} \cdot \vec{\sigma}) \times \mp^{\mathrm{a}}[\mathrm{b} \\
\\
\quad 2 \frac{W}{R}\left(\frac{W}{s}-1\right)^{2} W_{220} \\
+2 \frac{W}{R}\left(\frac{W}{s}-1\right)^{2} W_{222} \\
+3 \frac{W^{2}}{R^{2}}\left(\frac{W}{s}-1\right) W_{131} \\
\quad+4 \frac{W^{3}}{R^{3}} W_{040}\end{aligned}$ & $\begin{aligned} &\left(\frac{\vec{\rho}}{R} \cdot \frac{\vec{l}}{R}\right)(\vec{\sigma} \cdot \vec{\sigma}) \times[ \\
&-2\left(\frac{W}{s}-1\right)^{2} W_{220} \\
&-2\left(\frac{W}{s}\right)\left(\frac{W}{s}-1\right) W_{222} \\
&-2 \frac{W}{R}\left(\frac{3 W}{2 s}-1\right) W_{131} \\
&-4 \frac{W^{2}}{R^{2}} W_{040}\end{aligned}$ & $\begin{array}{l}\left(\frac{\vec{\rho}}{R} \cdot \vec{\alpha}\right)(\vec{\sigma} \cdot \vec{\sigma}) \times[ \\
-2 \frac{W}{R}\left(\frac{W}{s}-1\right) W_{222} \\
-\frac{W^{2}}{R^{2}} W_{131}\end{array}$ \\
\hline & & $\begin{aligned} &\left(\frac{\vec{\rho}}{R} \cdot \vec{\sigma}\right)\left(\vec{\sigma} \cdot \frac{\vec{l}}{R}\right) \times[ \\
&-4\left(\frac{W}{s}\right)\left(\frac{W}{s}-1\right) W_{220} \\
&-2\left(\frac{W}{s}-1\right)\left(\frac{2 W}{s}-1\right) W_{222} \\
&-4 \frac{W}{R}\left(\frac{3 W}{2 s}-1\right) W_{131} \\
&-8 \frac{W^{2}}{R^{2}} W_{040}\end{aligned}$ & $\begin{aligned}\left(\frac{\vec{\rho}}{R} \cdot \vec{\sigma}\right) & (\vec{\sigma} \cdot \vec{\alpha}) \times[ \\
& -4 \frac{W}{R}\left(\frac{W}{s}-1\right) W_{220} \\
& -2 \frac{W}{R}\left(\frac{W}{s}-1\right) W_{222} \\
& -2 \frac{W^{2}}{R^{2}} W_{131}\end{aligned}$ \\
\hline
\end{tabular}

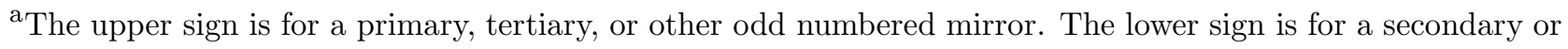
even numbered mirror.

${ }^{\mathrm{b}}$ The $W_{311}$ term has been omitted as it is equal to zero. 
are then due entirely to the decenter, $\vec{\ell}_{2}$ and tilt, $\vec{\alpha}_{2}$ of the secondary. One need only measure two of the five misalignment patterns in Table 2 to align the telescope. If the aperture stop (and consequently the pointing) instead coincide with the secondary mirror as in Noethe \& Guisard (2000), the misalignments of the secondary mirror may be treated as identically zero and only the misalignments of the primary mirror relative to its pupil need to be considered. In this case as well, one need only measure two of the five misalignment patterns in Table 2 to align the telescope. There is a third case where the aperture stop is independent from either of the mirrors. In this case we would treat the aperture stop as a third optical element and analyze this telescope configuration as a three mirror telescope.

McLeod (1996) measures coma and astigmatism in his two mirror telescope. He might in principle have used the two distortion patterns, but these would require a high precision astrometric catalog (perhaps using galaxy positions to avoid the effects of stellar proper motions). Moreover as the misalignment distortion patterns vary as a higher powers of field angle than the misalignment astigmatism and coma patterns, they might be expected to have smaller amplitudes. McLeod might also have used curvature of field, although here there is the danger that the detector might be tilted with respect to the primary. Or, had he been feeling particularly masochistic, he might have measured all five patterns, for the sake of redundancy.

With their folding flats, the Magellan telescopes in their Nasmyth configuration qualify as 2.5-mirror telescopes. One need not worry about the decentering of the tertiary but one must measure and correct for its tilt, $\vec{\alpha}_{3}$. The alignment procedure described by Palunas et al. (2010) adds the curvature of field misalignment pattern (equivalent to a focal plane tilt) to those of coma and astigmatism.

\section{Aligning 3-mirror telescopes using distortion patterns}

Calculating the aberration patterns for a 3-mirror telescope (say a three mirror anastigmat, henceforth a TMA) is not quite twice as difficult as for a 2-mirror telescope. One applies Table 2 to the tertiary and finds misalignment patterns that depend upon the decenter $\vec{\ell}_{3}$ and tilt $\vec{\alpha}_{3}$ of the tertiary. If the stop is coincident with the primary, only the secondary and tertiary contribute to the five third-order misalignment patterns in Table 2, The patterns are linear in the decenter and tilt vectors, so that the combined wavefront gives the same five patterns, each characterized by a new pattern 2 -vector. Each pattern 2 -vector, $\vec{\mu}$, is a linear combination of the four misalignment 2-vectors, the tilts $\vec{\alpha}_{2}$ and $\vec{\alpha}_{3}$, and the decenterings $\vec{\ell}_{2}$ and $\vec{\ell}_{3}$. Likewise if the stop is coincident with the secondary or tertiary mirrors, only tilts and decenters of the other two mirrors contribute. 
If coma, astigmatism and curvature of field are the only aberrations that adversely affect science, there is a two-dimensional "subspace of benign misalignment" for which the coma, astigmatism and curvature of field misalignment patterns are all zero in the limit of small misalignments. But if one does not bring additional information to bear, one runs the risk of drifting increasingly far from perfect alignment. This would produce large misalignment distortion, and ultimately, fifth-order misalignment patterns and third-order patterns that depend quadratically on the mirror tilts and decenters.

To measure the misalignment distortion patterns one would need either overlapping fields of images (Sudol 2011) or pre-existing astrometry. If one uses overlapping fields, one risks changing the misalignment between pointings, thus rendering distortion of limited use for measuring alignment at a given pointing. If one relies on pre-existing astrometry, the accuracy with which the distortion patterns could be measured would then be limited by the accuracy of the astrometric catalog. Moreover, regardless the technique used to measure the aberrations, the misalignment distortion patterns might be expected to have smaller amplitudes than misalignment astigmatism and coma as the misalignment distortion patterns vary as a higher power of field angle. This raises the question of whether one might use fifth-order aberration patterns to keep the telescope aligned.

\section{Generic fifth-order aberration patterns}

\section{1. fifth-order aberrations for a single mirror}

In $\$ 2.6$ we considered the third-order aberrations produced by a single mirror with a stop at the mirror. The generalization to fifth-order is straightforward. The fifth-order wavefront delay, $G^{5 t h}$ for a ray that hits the mirror at position $\vec{\varpi}$ and that makes an angle $\vec{\psi}$ with the axis of the mirror is given by

$$
\begin{aligned}
G^{5 t h} & =W_{060}\left(\frac{\vec{\varpi}}{R} \cdot \frac{\vec{\varpi}}{R}\right)\left(\frac{\vec{\varpi}}{R} \cdot \frac{\vec{\varpi}}{R}\right)\left(\frac{\vec{\varpi}}{R} \cdot \frac{\vec{\varpi}}{R}\right)+W_{151}\left(\frac{\vec{\varpi}}{R} \cdot \frac{\vec{\varpi}}{R}\right)\left(\frac{\vec{\varpi}}{R} \cdot \frac{\vec{\varpi}}{R}\right)\left(\frac{\vec{\varpi}}{R} \cdot \vec{\psi}\right) \\
& +W_{242}\left(\frac{\vec{\varpi}}{R} \cdot \frac{\vec{\varpi}}{R}\right)\left(\frac{\vec{\varpi}}{R} \cdot \vec{\psi}\right)\left(\frac{\vec{\varpi}}{R} \cdot \vec{\psi}\right)+W_{240}\left(\frac{\vec{\varpi}}{R} \cdot \frac{\vec{\varpi}}{R}\right)\left(\frac{\vec{\varpi}}{R} \cdot \frac{\vec{\varpi}}{R}\right)(\vec{\psi} \cdot \vec{\psi}) \\
& +W_{333}\left(\frac{\vec{\varpi}}{R} \cdot \vec{\psi}\right)\left(\frac{\vec{\varpi}}{R} \cdot \vec{\psi}\right)\left(\frac{\vec{\varpi}}{R} \cdot \vec{\psi}\right)+W_{331}\left(\frac{\vec{\varpi}}{R} \cdot \frac{\vec{\varpi}}{R}\right)\left(\frac{\vec{\varpi}}{R} \cdot \vec{\psi}\right)(\vec{\psi} \cdot \vec{\psi}) \\
& +W_{422}\left(\frac{\vec{\varpi}}{R} \cdot \vec{\psi}\right)\left(\frac{\vec{\varpi}}{R} \cdot \vec{\psi}\right)(\vec{\psi} \cdot \vec{\psi})+W_{420}\left(\frac{\vec{\varpi}}{R} \cdot \frac{\vec{\varpi}}{R}\right)(\vec{\psi} \cdot \vec{\psi})(\vec{\psi} \cdot \vec{\psi}) \\
& +W_{511}\left(\frac{\vec{\varpi}}{R} \cdot \vec{\psi}\right)(\vec{\psi} \cdot \vec{\psi})(\vec{\psi} \cdot \vec{\psi})
\end{aligned}
$$


Since the pupil is coincident with the mirror, we might equally well have written the same equations but with position on the mirror $\vec{\varpi}$ replaced by position on the pupil $\vec{\rho}$ and the angle that a ray makes with the axis of the mirror, $\vec{\psi}$ replaced by the field angle $\vec{\sigma}$. For the remainder of this subsection we shall take $\vec{\rho}=\vec{\varpi}$ and $\vec{\sigma}=\vec{\psi}$.

\subsection{1. $W_{511}$ : fifth-order distortion}

The $W_{511}$ term has the same variation on the pupil as distortion, but varies as field angle to the fifth. We shall refer to this as "fifth-order distortion." 8

\subsection{2. $W_{420}$ : fifth-order defocus}

The $W_{420}$ term has the same variation on the pupil as defocus, and produces a point spread function indistinguishable from that of Seidel curvature of field. But as this term varies as the fourth power of field angle rather than quadratically, we call this term "fifthorder defocus."

\subsection{3. $W_{422}$ : fifth-order astigmatism}

The $W_{422}$ term has the same variation on the pupil as third-order astigmatism, and produces a point spread function indistinguishable from it. But as this term varies as the fourth power of field angle rather than quadratically, we call this term "fifth-order astigmatism." Again as with third-order astigmatism, this term varies on the pupil as $\rho^{2} \cos ^{2} \phi$. It can be decomposed into a term that varies as Zernike astigmatism, $\rho^{2} \cos 2 \phi$, and a second that varies as defocus, $\rho^{2} \cos ^{0} \phi$,

\footnotetext{
${ }^{8}$ Our nomenclature is driven primarily by the functional form of the aberration on the pupil. Thus an aberration that varies as $\rho^{3} \cos \phi$ is referred to as coma. In this scheme third-order (Seidel) coma varies linearly with field angle and fifth-order coma varies as the cube of field angle. By contrast, the term that varies as $\rho^{5} \cos \phi$ on the pupil is referred to here as second coma or coma-II. Hopkins (1950) calls this term fifth-order coma, but from our pupil oriented perspective this term, albeit one of fifth-order, cannot be called coma, which can only vary as $\rho^{3} \cos \phi$. Curiously, we agree with Hopkins in calling the term that varies as $\rho^{2} \cos ^{2} \phi$ fifth-order astigmatism and in calling the term that varies as $\rho \cos \phi$ fifth-order distortion. We reserve the term "Zernike" aberrations for the orthogonalized linear combinations of aberrations described here.
} 


\subsection{4. $W_{331}$ : fifth-order coma}

The $W_{331}$ term has the same variation on the pupil as coma, but varies as field angle cubed. We call this "fifth-order coma."

\subsection{5. $W_{333}$ : trefoil}
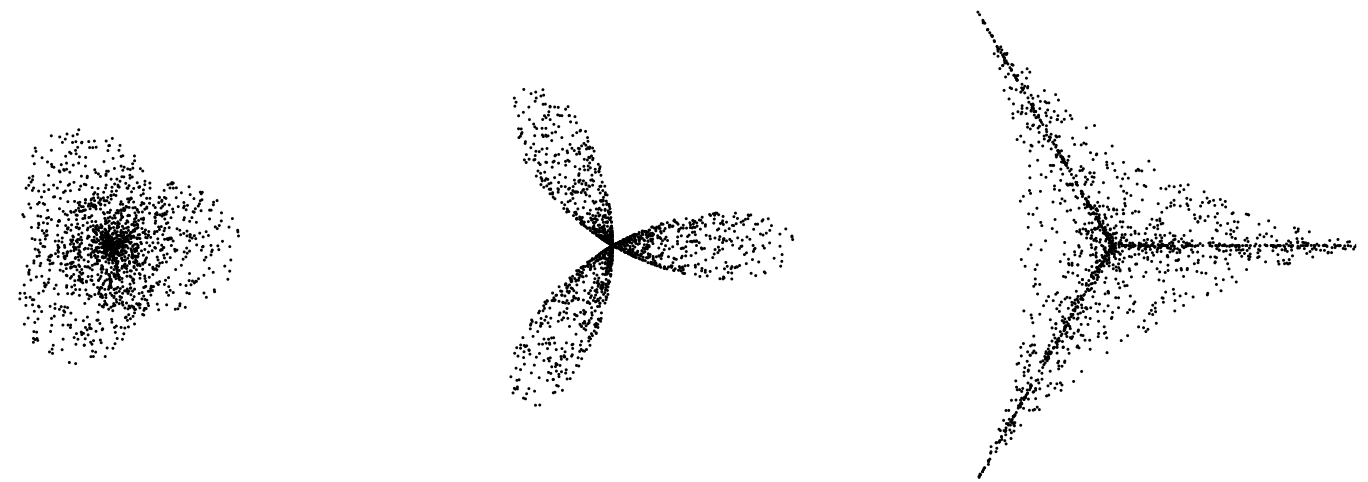

Fig. 5.- Point spread functions due to trefoil with $G^{\text {tref }}=\rho^{3} \cos 3 \phi$. Increasing amounts of a symmetric wavefront $G^{\text {sym }}=\rho^{3}$ have been added to bring out the three-fold symmetry of the wavefront. (a) $+\frac{2}{5} G^{\text {sym }}(\mathrm{b})+G^{\text {sym }}(\mathrm{c})+2 G^{\text {sym }}$

The $W_{333}$ term varies as $\rho^{3} \cos ^{3} \phi$ on the pupil and as field angle cubed. None of the third-order aberrations has this behavior on the pupil; here we call it trefoil. This term can be decomposed into a term that varies as Zernike trefoil, $\rho^{3} \cos 3 \phi$, and a second that varies as coma, $\rho^{3} \cos \phi$. Figure 5 shows the point spread function due to Zernike trefoil with varying amounts of an aberration that varies as $\rho^{3}$ added to it.

\subsection{6. $W_{240}$ : fifth-order spherical}

The $W_{240}$ has the same variation on the pupil as spherical aberration, but varies as field angle squared. We shall refer to this as this "fifth-order spherical." 

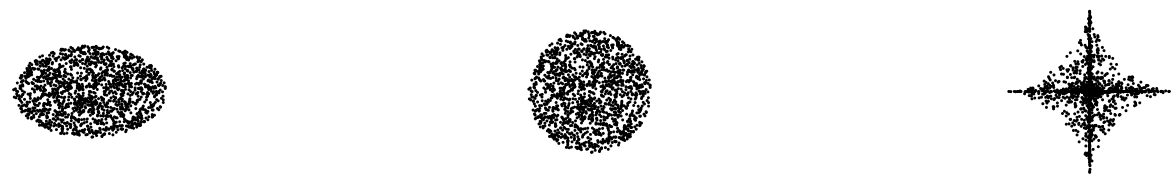

Fig. 6.- Point spread functions for ordinary astigmatism, $G^{a s t i g-I}=\rho^{2} \cos 2 \phi$ and second astigmatism, $G^{\text {astig-II }}=\rho^{4} \cos 2 \phi$. (a) Ordinary astigmatism $+\frac{1}{4} G^{\text {defocus }}$. (b) Pure ordinary astigmatism. (c) Pure second astigmatism.

\subsection{7. $W_{242}$ : second astigmatism}

The $W_{242}$ term varies as $\rho^{4} \cos ^{2} \phi$ on the pupil. The angular dependence is that of astigmatism but the radial dependence is quartic not quadratic. We shall call this "second astigmatism" or "astigmatism-II." Figure 6] shows the PSF for second astigmatism next to those for ordinary astigmatism.

\subsection{8. $W_{151}$ : second coma}

The $W_{151}$ term varies as $\rho^{5} \cos \phi$ on the pupil. The azimuthal dependence is that of coma but the radial dependence is quintic not cubic. We shall refer to this as "second coma" or "coma-II." Figure 7 shows a PSF produced by second coma alongside one produced by ordinary (first) coma.

\subsection{9. $W_{060}:$ second spherical}

We shall call the $W_{060}$ term "second spherical" or "spherical-II." Were we strictly consistent we would have called spherical aberration "second defocus" and would have called this "third defocus" but as Emerson says, a foolish consistency is the hobgoblin of a small mind. 

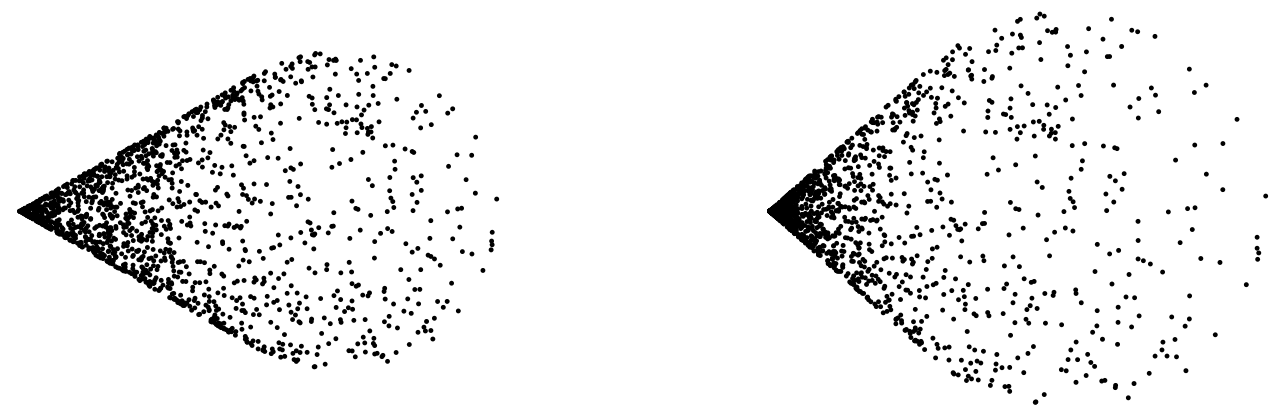

Fig. 7.- (a) Off axis (ordinary) coma PSF for the third-order and fifth-order coma patterns. The sine of the apex half angle is $1 / 2$, giving an apex angle of $60^{\circ}$. (b) Off axis second coma PSF. The sine of the apex half angle is $2 / 3$, giving an apex angle of $83.6^{\circ}$.

\section{2. fifth-order aberrations for misaligned systems}

Tilts and decenterings of mirrors produce misalignment patterns in a manner entirely analogous to those of third-order aberrations. The dependence upon field and pupil position and the first order dependence on misalignments are given in Table 3 , Equations (66) and (7) are used to transform from the positions and angles with respect to the optic, $\vec{\varpi}$ and $\vec{\psi}$ of equation (8) to positions and angles with respect the pupil, $\vec{\rho}$ and $\vec{\sigma} \cdot 9$ Terms with the same dependence on both of the latter are then added. The vectors $\vec{\mu}$ indicate linear combinations of misalignment angle $\vec{\alpha}$ and decenter $\vec{\ell}$.

In Table 3 we give only the dependence upon pupil position and field angle for the symmetric fifth-order aberration patterns and their associated misalignment patterns. For the third-order aberrations, there was one misalignment pattern each associated with coma, astigmatism and curvature of field, with two misalignment patterns associated with distor-

\footnotetext{
${ }^{9}$ Third-order aberrations produced by an optic (equation (5)) may also contribute to the fifth-order aberrations on the pupil when the pupil and the optic do not coincide. As noted in $\$ \$ 2.6$ equations (6) and (17) are truncated at first order in pupil and field coordinates. Analogous equations for (6) and (77) that have been expanded to third order can map third-order aberrations on the optic to fifth-order aberrations on the pupil. Other such approximations may also need to be relaxed in order to compute the magnitudes of the fifth-order aberrations.
} 
tion. There are again distinct $\sigma$ and $\rho$ misalignment patterns for fifth-order distortion, and now also for fifth-order astigmatism and fifth-order coma.

\section{3. fifth-order aberration patterns and alignment}

The amplitudes of aberration patterns implicit in Table 3 provide, at least in principle, additional information for use in aligning telescopes. But in all cases there are similarities between the fifth-order misalignment aberration patterns and their third-order counterparts. The ability to distinguish between the two depends, for the first group described below, upon the sampling of the wavefront, and, for the second group, upon the sampling of the field.

\subsection{1. second coma: misalignment}

The misalignment aberration pattern for second coma, shown in Figure 8 is identical to that for ordinary (first) coma (see Figure1). The point spread functions for second coma and ordinary (first) coma have the same azimuthal dependence on pupil position but different radial dependence. The ability to distinguish between the coma-II misalignment pattern and the coma-I misalignment pattern therefore depends critically upon the sampling of the wavefront.

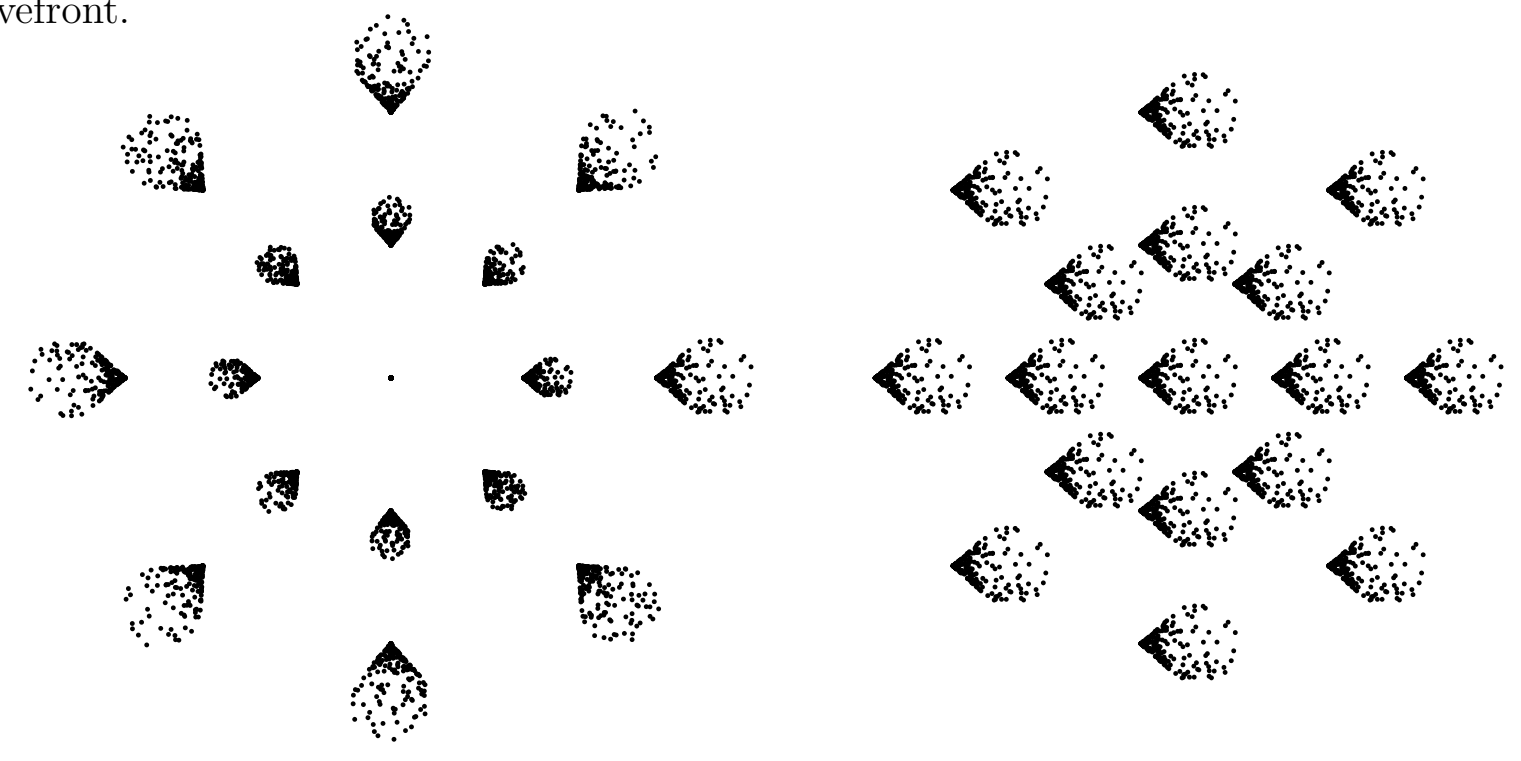

Fig. 8. - Second coma field patterns. (a) Second coma pattern typical of an aligned telescope. (b) Second coma pattern indicating a tilt and (or) decenter of a mirror along the $\mathrm{x}$ axis. 


\subsection{2. second astigmatism: misalignment}
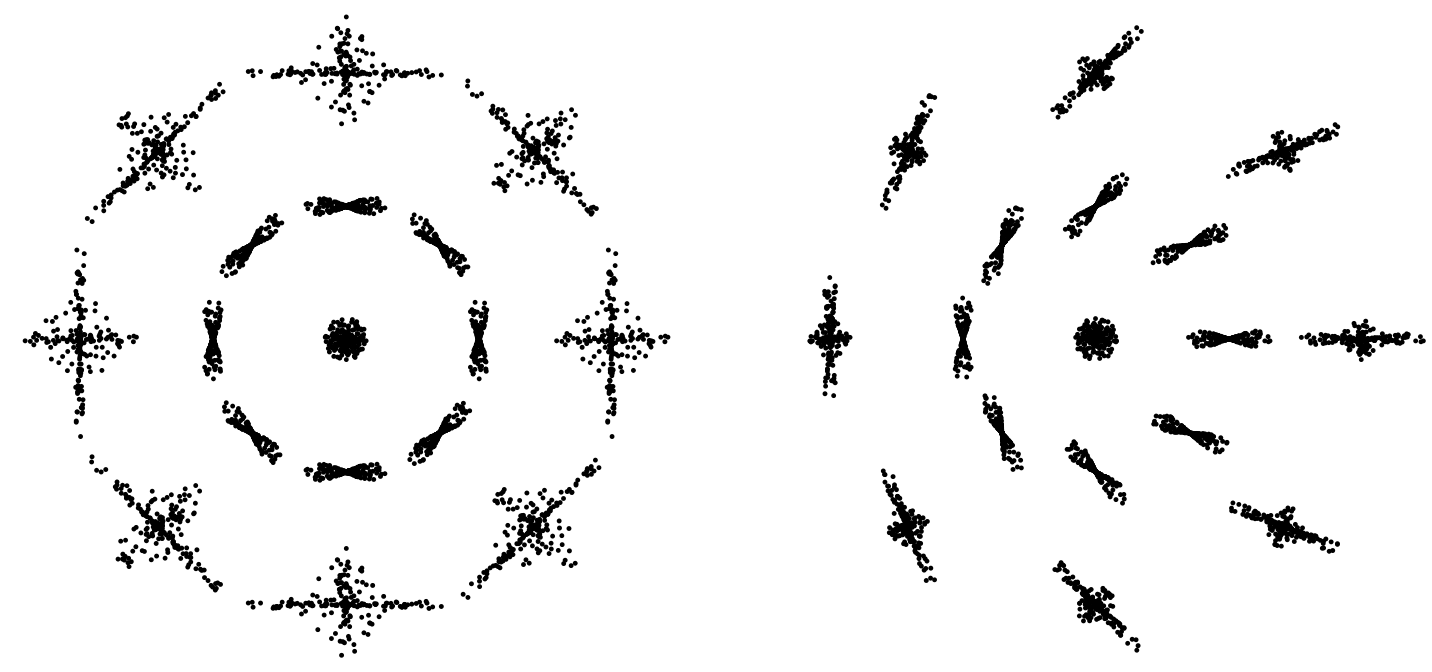

Fig. 9.- Second astigmatism field patterns. (a) Second astigmatism pattern typical of an aligned telescope. (b) Second astigmatism pattern indicating a tilt and (or) decentering of a mirror along the $\mathrm{x}$ axis. Spherical aberration has been added to bring out the asymmetry of the pattern.

As with second coma, the misalignment aberration pattern for second astigmatism, shown in Figure 9 is identical to that for ordinary (first) astigmatism (see Figure 21). The point spread functions for second astigmatism and ordinary (first) astigmatism have the same angular dependence on pupil position but different radial dependence. The ability to distinguish between the astigmatism-II misalignment pattern and the astigmatism-I misalignment pattern again depends critically upon the sampling of the wavefront.

\subsection{3. fifth-order spherical: misalignment}

The aberration patterns for fifth-order spherical, shown in Figure 10 are identical to those for those of curvature of field. However the point spread functions are identical to spherical aberration, which itself has the same angular dependence on the pupil as defocus and COF, but a different radial dependence. The ability to distinguish between the fifthorder spherical and curvature of field misalignment pattern once again depends critically upon the sampling of the wavefront. The ability to distinguish between fifth-order spherical and ordinary spherical depends on the sampling of the field. 
Table 3. Fifth-order aberration patterns

\begin{tabular}{lll}
\hline \hline \multicolumn{1}{c}{ aberration } & \multicolumn{1}{c}{ symmetric } & \multicolumn{1}{c}{ misalignment } \\
\hline 2nd spherical & $(\vec{\rho} \cdot \vec{\rho})(\vec{\rho} \cdot \vec{\rho})(\vec{\rho} \cdot \vec{\rho})$ & \\
2nd coma & $(\vec{\rho} \cdot \vec{\rho})(\vec{\rho} \cdot \vec{\rho})(\vec{\rho} \cdot \vec{\sigma})$ & $(\vec{\rho} \cdot \vec{\rho})(\vec{\rho} \cdot \vec{\rho})\left(\vec{\rho} \cdot \vec{\mu}_{c I I}\right)$ \\
2nd astigmatism & $(\vec{\rho} \cdot \vec{\rho})(\vec{\rho} \cdot \vec{\sigma})(\vec{\rho} \cdot \vec{\sigma})$ & $(\vec{\rho} \cdot \vec{\rho})(\vec{\rho} \cdot \vec{\sigma})\left(\vec{\rho} \cdot \vec{\mu}_{a I I}\right)$ \\
fifth-order spherical & $(\vec{\rho} \cdot \vec{\rho})(\vec{\rho} \cdot \vec{\rho})(\vec{\sigma} \cdot \vec{\sigma})$ & $(\vec{\rho} \cdot \vec{\rho})(\vec{\rho} \cdot \vec{\rho})\left(\vec{\sigma} \cdot \vec{\mu}_{5 s}\right)$ \\
trefoil & $(\vec{\rho} \cdot \vec{\sigma})(\vec{\rho} \cdot \vec{\sigma})(\vec{\rho} \cdot \vec{\sigma})$ & $(\vec{\rho} \cdot \vec{\sigma})(\vec{\rho} \cdot \vec{\sigma})\left(\vec{\rho} \cdot \vec{\mu}_{t}\right)$ \\
fifth-order coma & $(\vec{\rho} \cdot \vec{\rho})(\vec{\rho} \cdot \vec{\sigma})(\vec{\sigma} \cdot \vec{\sigma})$ & $(\vec{\rho} \cdot \vec{\rho})(\vec{\rho} \cdot \vec{\sigma})\left(\vec{\sigma} \cdot \vec{\mu}_{5 c \sigma}\right)$ \\
& & $(\vec{\rho} \cdot \vec{\rho})(\vec{\sigma} \cdot \vec{\sigma})\left(\vec{\rho} \cdot \vec{\mu}_{5 c \rho}\right)$ \\
fifth-order astigmatism & $(\vec{\rho} \cdot \vec{\sigma})(\vec{\rho} \cdot \vec{\sigma})(\vec{\sigma} \cdot \vec{\sigma})$ & $(\vec{\rho} \cdot \vec{\sigma})(\vec{\rho} \cdot \vec{\sigma})\left(\vec{\sigma} \cdot \vec{\mu}_{5 a \sigma}\right)$ \\
& & $(\vec{\rho} \cdot \vec{\sigma})(\vec{\sigma} \cdot \vec{\sigma})\left(\vec{\rho} \cdot \vec{\mu}_{5 a \rho}\right)$ \\
fifth-order defocus & $(\vec{\rho} \cdot \vec{\rho})(\vec{\sigma} \cdot \vec{\sigma})(\vec{\sigma} \cdot \vec{\sigma})$ & $(\vec{\rho} \cdot \vec{\rho})(\vec{\sigma} \cdot \vec{\sigma})\left(\vec{\sigma} \cdot \vec{\mu}_{5 f}\right)$ \\
fifth-order distortion & $(\vec{\rho} \cdot \vec{\sigma})(\vec{\sigma} \cdot \vec{\sigma})(\vec{\sigma} \cdot \vec{\sigma})$ & $(\vec{\rho} \cdot \vec{\sigma})(\vec{\sigma} \cdot \vec{\sigma})\left(\vec{\sigma} \cdot \vec{\mu}_{\left.5 d_{\sigma}\right)}\right)$ \\
& & $(\vec{\sigma} \cdot \vec{\sigma})(\vec{\sigma} \cdot \vec{\sigma})\left(\vec{\rho} \cdot \vec{\mu}_{5 d \rho}\right)$ \\
\hline
\end{tabular}

Fig. 10.- Fifth-order spherical field patterns. (a) Spherical aberration pattern typical of an aligned telescope. (b) Spherical aberration indicating a tilt and (or) decenter of a mirror along the $\mathrm{x}$ axis. 


\subsection{4. trefoil: misalignment}
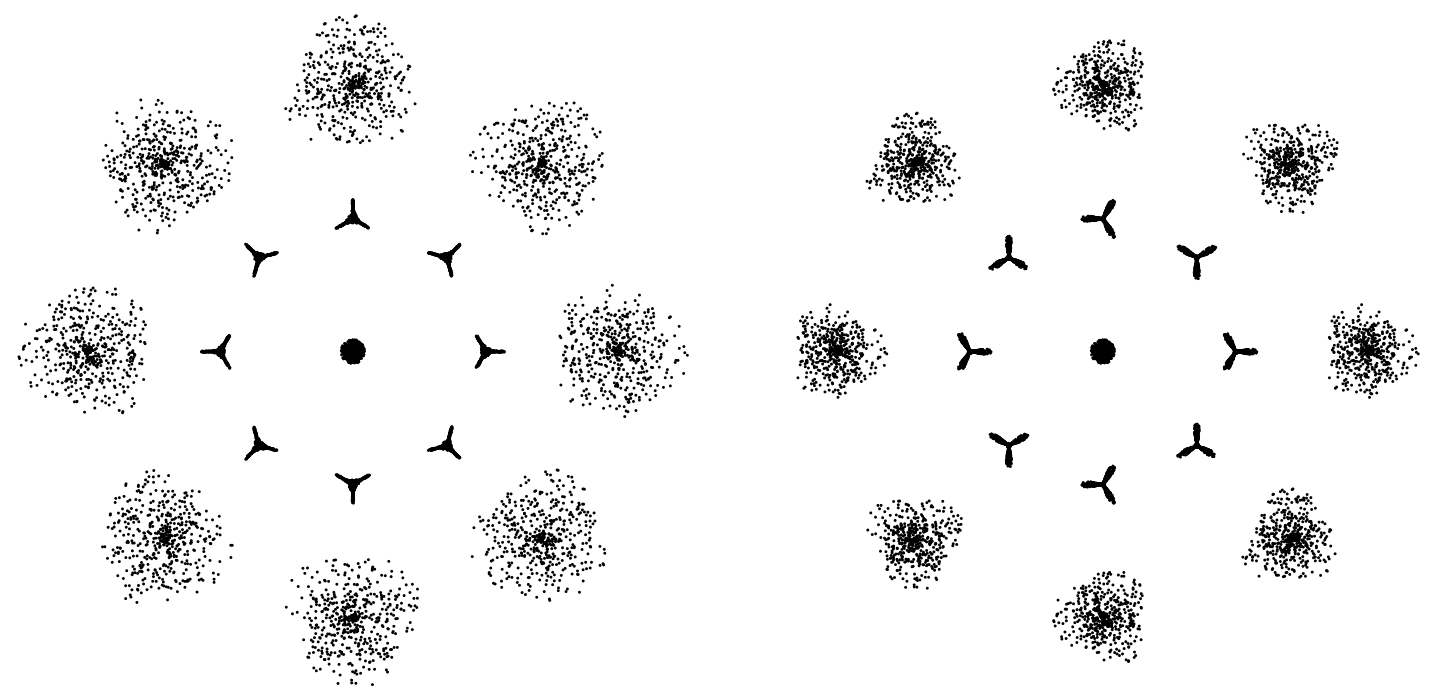

Fig. 11. - Trefoil field patterns. (a) Trefoil pattern characteristic of an aligned telescope. (b) Trefoil indicating a tilt and (or) decenter of a mirror along the $\mathrm{x}$ axis. In both cases a constant wavefront varying as $\rho^{3}$ has been added to bring out the orientation of the aberration.

The PSF for trefoil is quite different from that of any of the Seidel third-order aberrations, as is its misalignment pattern, shown in Figure 11.

\subsection{5. fifth-order astigmatism: misalignment}

The PSF for fifth-order astigmatism is identical to that of Seidel, third-order astigmatism. But the two misalignment aberration patterns shown in Figure 12 are unlike thirdorder misalignment astigmatism (see Figure 21). The $\rho$ misalignment pattern has the same azimuthal field dependence as for third-order astigmatism, but has a different radial dependence. The $\sigma$ misalignment pattern has a different azimuthal field dependence and a different radial dependence. The ability to distinguish between the two fifth-order astigmatism misalignment patterns and the third-order astigmatism misalignment pattern therefore depends critically upon the sampling of the field rather than the wavefront. 

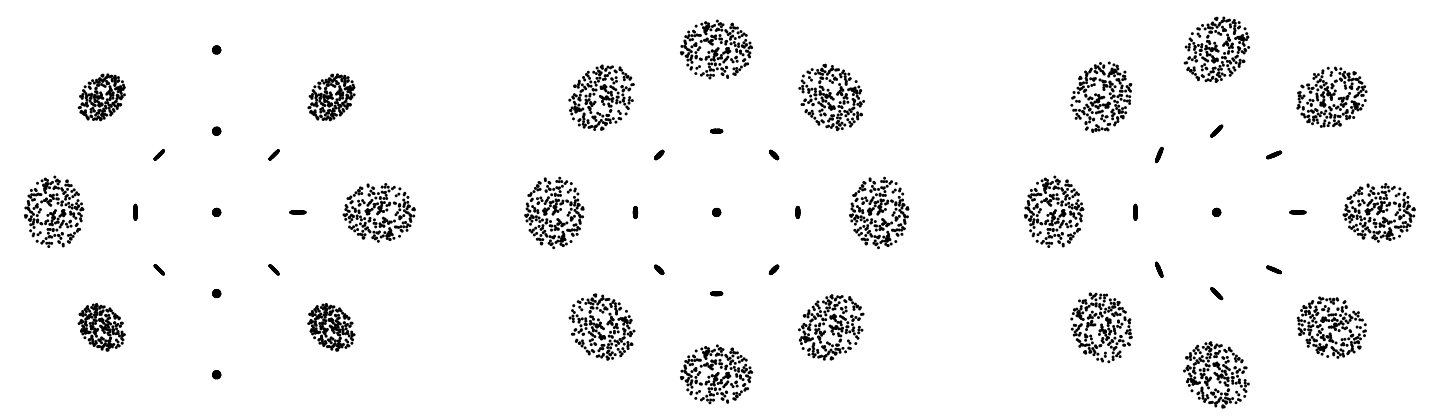

Fig. 12.- Fifth-order astigmatism field patterns. (a) The " $\sigma$ " astigmatism pattern indicating a tilt and (or) decentering of a mirror along the $\mathrm{x}$ axis. Note that the astigmatism is radially aligned. (b) Astigmatism pattern typical of an aligned telescope. (c) The " $\rho$ " astigmatism pattern indicating a tilt and (or) decentering of a mirror along the $\mathrm{x}$ axis. Note that the azimuthal dependence on field angle is identical to that for third-order misalignment astigmatism. A constant defocus has been added to all three panels to show the orientations of the aberrations.
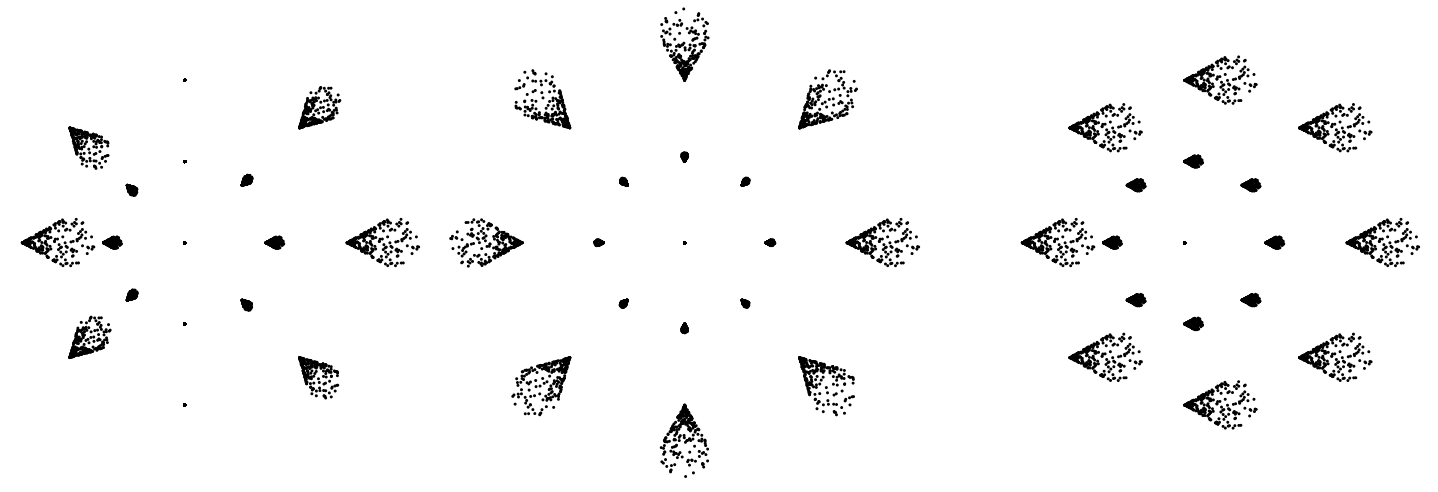

Fig. 13. - Fifth-order coma field patterns. (a) The " $\sigma$ " coma pattern indicating a tilt and (or) decentering of a mirror along the $\mathrm{x}$ axis. Note that the coma is radially aligned. (b) Fifth-order coma characteristic of an aligned telescope (c) The " $\rho$ " coma pattern indicating a tilt and (or) decentering of a mirror along the $\mathrm{x}$ axis. Note that the azimuthal dependence on field angle is identical to that for third-order misalignment coma. 


\subsection{6. fifth-order coma: misalignment}

As with fifth-order astigmatism, the PSF for fifth-order coma is identical to that of Seidel, third-order coma. But the two misalignment aberration patterns shown in Figure 13 are unlike third-order misalignment coma (see Figure11). The $\rho$ misalignment pattern has the same azimuthal field dependence as for third-order coma, but different radial dependence. The $\sigma$ misalignment pattern has a different azimuthal field dependence and a different radial dependence. As with fifth-order astigmatism, the ability to distinguish between the two fifthorder coma misalignment patterns and the third-order coma misalignment pattern therefore depends critically upon the sampling of the field rather than the wavefront.

\subsection{7. fifth-order defocus: misalignment}

As with fifth-order astigmatism and coma, the PSF for fifth-order defocus is identical to that of curvature of field (which might equally well be called third-order defocus). But the misalignment aberration patterns are not identical. They have the same angular dependence, but exhibit a different radial dependence. Once again, the ability to distinguish between the fifth-order defocus misalignment pattern and the curvature of field misalignment pattern depends critically upon the sampling of the field rather than the wavefront.

\section{4. discussion of fifth-order misalignment aberration patterns}

The literature on fifth-order aberrations is limited for several reasons. First, by their

very nature, they tend to be smaller than the third-order (Seidel) aberrations. Second, they are rather cumbersome. As a matter of course, ray tracing programs handle them correctly, so as a matter of practice, they receive little attention.

But as we have seen in the previous section, fifth-order aberrations may be needed to keep a three-mirror telescope aligned, and would almost certainly be needed to keep a four-mirror telescope aligned. Conversely, if one uses only the more commonly measured third-order aberrations, coma, astigmatism, and COF, to keep a telescope aligned, there are degenerate telescope configurations that zero out these third-order misalignment aberrations and yet produce distortion and fifth-order misalignment aberrations. It is therefore of some interest to estimate which of these might be more or less substantial.

An order of magnitude argument can be made by noting that the entries in Table 3 are homogeneous and of sixth order in the sum of the exponents of the pupil radius and the 
field angle. Both of these are rendered dimensionless by the focal lengths. We would argue that it is the focal ratio of the fastest element that matters most in such considerations. Modern fast, wide field telescopes have primary f-ratios approaching unity, while the field angles are rarely greater than a tenth of a radian. By this argument the terms at the top of Table 3 would be larger than those at the bottom. Third- and fifth-order aberrations for three specific optical systems: the LSST, HET corrector, and a Magellan-like telescope, are analyzed in the following subsections.

\subsection{Tessieres' models for the LSST}

The approach advocated here is quite similar to that adopted by Regis Tessieres in an unpublished M.S. thesis (2003) carried out at the University of Arizona. Tessieres analyzed the off-axis aberrations for two telescopes in terms of patterns derived in Thompson's unpublished (1980) PhD thesis. But instead of computing the amplitudes of the patterns according to the principles set forth by Thompson, he used ray-tracing software to produce wavefronts across the field for various misalignments. He then fit these wavefronts to the expected patterns.

Of particular relevance for the present work, he analyzed an early version of the Large Synoptic Survey Telescope (Seppala 2002; henceforth LSST) in which the tertiary and primary were independent (rather than fabricated from a single monolith, as with the ultimate design). He applied decenters and tilts to the secondary and tertiary (and to the corrector assembly) and decomposed the computed wavefronts into the third- and fifth-order aberration patterns. Results of those calculations are given in Table 4, which show the effects of tilts in the secondary and tertiary 10 Each entry gives the amplitude of the aberration at the edge of the field and at the edge of the pupil, in microns, for one degree of tilt. Up to factors of order unity the rms spot size will be proportional to these.

The first impression one gets is that the third-order aberrations are factors of 30-300 larger than the fifth-order aberrations. A consequence of this disparity is that, to the extent that the fifth-order and third-order aberration patterns are correlated, a small relative error in a measurement of a third-order aberration pattern will produce a large relative error in the corresponding fifth-order aberration pattern. This bodes ill for using fifth-order aberrations for telescope alignment. Tessieres' calculations are themselves not entirely immune from such errors, but Tessieres had the luxury of measuring the wavefront with high precision at

\footnotetext{
10 Tessieres' nomenclature is similar to Hopkins', but each aberration is preceded by its field angle dependence. Thus what we would called third-order misalignment astigmatism he calls field-linear astigmatism.
} 
a large number of points in the field.

There are several patterns for which Tessieres did not report amplitudes - fifth-order astigmatism- $\sigma$ and the $\rho$ patterns for fifth-order coma and astigmatism for the secondary mirror. One suspects that the amplitudes for these were so small as to be in the noise, but Tessieres is not explicit on this point.

Tessieres did fit several patterns that vary quadratically with tilt and decenter. We suspect that the coefficients for these would approach zero for successively smaller tilts and decenters. Assuming iterative alignment correction these will play no role once the misalignments are small, and we have not included them in our discussion.

Tessieres did not measure the misalignment patterns for either spherical aberration or curvature of field (defocus). These surely contributed to his figure of merit, and his alignment experiments might have converged more rapidly had he measured them.

Finally Tessieres did not measure distortion, which might in principle be used to align a telescope.

\subsection{Manuel's models for the HET corrector}

In another unpublished Ph.D. thesis, Anastacia Manuel (2009), working at the University of Arizona, carried out a ray-tracing misalignment analysis of a four-element corrector for the Hobby-Ebberly telescope.

The emphasis was on identifying the combinations of motions of the four elements that produced the largest aberrations using singular value decomposition. These modes sometimes involved more than one of the patterns considered here. Several of the larger modes were associated with despacing and manifested themselves in symmetric aberration patterns.

But of the misalignment patterns, the three largest were the misalignments associated coma, curvature of field, and astigmatism, all of which are third-order. Next after that came linear combinations of second coma and second astigmatism misalignment patterns. These modes produced a figure of merit (which we take to be proportional to the wavefront error) a factor of $10^{4}$ smaller than those for coma-I and a factor of 30 smaller than those for astigmatism-I. This would again suggest that measurements of fifth-order aberrations may not contribute much to aligning the system in question. 


\section{7. modeled aberrations for a Magellan-like telescope}

In order to explore the feasibility of using distortion or the fifth-order aberrations for telescope alignment, we used Zemax ${ }^{\circledR}$ to measure the third- and fifth-order aberrations of a Gregorian telescope adapted from the Magellan Baade and Clay telescopes. The model telescope consists of three optical elements and a detector: a $6.4784 \mathrm{~m}$ diameter aperture stop which sits $0.32512 \mathrm{~m}$ in front of the primary mirror, a primary mirror with radius of curvature of $-16.2553 \mathrm{~m}$ and conic constant -1.00001 , and a secondary mirror $9.72205 \mathrm{~m}$ in front of the primary with radius of curvature $2.86282 \mathrm{~m}$ and conic constant -0.63286 . The focal plane sits $4.28026 \mathrm{~m}$ behind the primary mirror. We analyzed two telescope configurations: one with a $1 \mathrm{~mm}$ decentered secondary mirror and one with a $1 / 4^{\circ}$ tilted secondary mirror. The telescope specs appear in table 5 .

For each telescope configuration, the Zemax ${ }^{\circledR}$ analysis tool Fringe Zernike was used to compute the Zernike wavefront aberrations at the edge of the pupil for a total of 21 field points spanning the $x$ and $y$ axes of the field. For the points along each telescope axis, the Zernikes

corresponding to the pupil variations given in tables 2 and 3 were then fit to the field patterns using a simple least squares algorithm. Measurement of the wavefront delay along two field axes was necessary to distinguish the effects of $\rho$ and $\sigma$ fifth-order astigmatism and also $\rho$ and $\sigma$ fifth-order coma. For the other aberrations the second field axis provided a redundancy with which to verify the results. All of those third-order patterns fitted redundantly are selfconsistent to within $0.2 \%$. The fifth-order patterns are self-consistent to within $2 \%$ with the exception of second coma for the tilted telescope and misalignment trefoil for the decentered telescope. The former is likely corrupted by higher order effects which will be discussed later, and the latter is consistent with zero.

We also used the Zemax ${ }^{\circledR}$ analysis tool Grid Distortion to analyze third- and fifthorder distortion for both telescope configurations. As with fifth-order coma and fifth-order astigmatism, we fit the distortion and fifth-order distortion patterns along two separate field axes in order to distinguish between the effects of the $\rho$ and $\sigma$ misalignment patterns.

The fitted aberrations for third- and fifth-order and the corresponding analytically computed aberrations for third-order appear in table 6. The coefficients are given in microns of wavefront delay at the edge of the pupil for an image at the edge of a $1^{\circ}$ field, with the exception of distortion and fifth-order distortion which are given in arcseconds of image displacement at the edge of the field.

While the magnitude of misalignment aberrations are specific to telescope design, for the Magellan-type telescope modeled here, the fifth-order misalignment aberrations are never more than $4 \%$ of the magnitude of the third-order misalignment coma aberration (and more 
Table 4. Amplitude of misalignment patterns at edge of the field for an early version of the LSST (in microns) for tilts of $1^{\circ}$.

\begin{tabular}{llll}
\hline \hline \multicolumn{1}{c}{ here } & \multicolumn{1}{c}{ Tessieres } & M2 & M3 \\
\hline third-order coma & constant coma & 178 & 186 \\
third-order astigmatism & linear astigmatism & 114 & 36 \\
coma-II & constant fifth-order coma & 2.4 & 4.3 \\
astigmatism-II & linear oblique spherical & 0.58 & 0.17 \\
trefoil & quadratic elliptical coma & 0.41 & 0.49 \\
fifth-order coma- $\sigma$ & quadratic coma\#2 & 0.90 & 2.09 \\
fifth-order coma- $\rho$ & quadratic coma\#1 & $a$ & 3.5 \\
fifth-order astigmatism- $\rho$ & cubic astigmatism\#1 & $a$ & 1.72 \\
\hline
\end{tabular}

${ }^{a}$ no value given

Table 5. Design specifications of the Magellan-like telescope.

\begin{tabular}{lcccc}
\hline \hline \multicolumn{1}{c}{ Surface } & $\begin{array}{c}\text { Radius of } \\
\text { Curvature }(\mathrm{m})\end{array}$ & Diameter $^{\mathrm{a}}(\mathrm{m})$ & Conic Constant & $\begin{array}{c}\text { Distance }(\mathrm{m}) \text { to } \\
\text { Next Surface }\end{array}$ \\
\hline object plane & - & - & - & infinity \\
aperture stop & - & 6.4784 & - & 0.32512000 \\
primary mirror & -16.2553 & 6.4805 & -1.00001 & -9.72205034 \\
secondary mirror & 2.86282 & 1.6134 & -0.63286 & 14.00230590 \\
image plane & - & - & - & - \\
\hline
\end{tabular}

${ }^{a}$ Diameter of the optic is larger than the diameter of the beam at the optic. 
Table 6. Amplitudes of aberration patterns for a Magellan-like telescope.

\begin{tabular}{|c|c|c|c|}
\hline Aberration & Symmetric & $\begin{array}{l}\text { Misalignment }(\rho, \sigma) \\
\text { Decentered } 1 \mathrm{~mm}\end{array}$ & $\begin{array}{c}\text { Misalignment } \\
\text { Tilted } 1 / 4^{\circ}\end{array}$ \\
\hline \multicolumn{4}{|c|}{ Third-order } \\
\hline spherical & -0.005 & & \\
\hline coma & -29.161 & -15.600 & 108.527 \\
\hline astigmatism & 129.592 & -0.479 & 51.848 \\
\hline $\mathrm{COF}$ & -591.745 & 7.631 & -72.386 \\
\hline \multirow{3}{*}{ distortion $^{\mathrm{a}}$} & -7.908 & 0.079 & -1.363 \\
\hline & & 0.098 & -0.619 \\
\hline & \multicolumn{2}{|c|}{ Fifth-order } & \\
\hline spherical-II & 0.000 & & \\
\hline coma-II & 0.013 & 0.587 & -4.087 \\
\hline astigmatism-II & 1.005 & -0.014 & 0.031 \\
\hline fifth-order spherical & -0.592 & 0.039 & -0.248 \\
\hline trefoil & 0.261 & -0.001 & 0.263 \\
\hline \multirow[t]{2}{*}{ fifth-order coma } & 3.641 & -0.037 & -0.375 \\
\hline & & -0.055 & 0.286 \\
\hline \multirow[t]{2}{*}{ fifth-order astigmatism } & -0.031 & 0.001 & -0.143 \\
\hline & & -0.003 & 0.280 \\
\hline fifth-order defocus & 0.102 & 0.002 & -0.168 \\
\hline \multirow[t]{2}{*}{ fifth-order distortion ${ }^{\mathrm{a}}$} & 0.010 & 0.000 & -0.003 \\
\hline & & 0.024 & 0.027 \\
\hline \multicolumn{4}{|c|}{ Analytic third-order } \\
\hline spherical & -0.005 & & \\
\hline coma & -29.180 & -15.625 & 108.702 \\
\hline astigmatism & 129.731 & -0.425 & 51.485 \\
\hline $\mathrm{COF}$ & -591.864 & 7.660 & -29.027 \\
\hline \multirow[t]{2}{*}{ distortion $^{\mathrm{a}}$} & -7.787 & 0.078 & -1.369 \\
\hline & & 0.112 & -0.875 \\
\hline
\end{tabular}

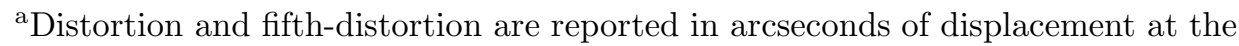
edge of a $1^{\circ}$ field. All other values are reported in microns of wavefront delay at the edge of the pupil. 
often than not, less than $1 \%$ of misalignment coma). While the misalignment distortion terms are also small, $-1^{\prime \prime} .363$ and $-0^{\prime \prime} .619$ for $\rho$ and $\sigma$ distortion respectively in the tilted telescope, they are significant compared to the reported RMS error of the SDSS astrometric catalog, 0 . $045-0$ '.075 and 0 '.100 for stars with magnitudes up to 20 and 22 (Pier et al. 2003). If the wavefront sensors on this hypothetical telescope sample the pupil well, second coma is the most easily measured fifth-order aberration with which to align the secondary. If field sampling is preferable to pupil sampling and either a catalog exists for the field or one is willing to dither on the field (Sudol 2011), distortion could be used for alignment.

In general, there is good agreement between the magnitudes of the third-order aberrations computed analytically using the formulae presented here and by Zemax ${ }^{\circledR}$. By far the most significant discrepancy between the analytically computed aberrations and those measured by Zemax ${ }^{\circledR}$ is for misalignment curvature of field for the tilted mirror. This discrepancy could be due to an inadvertent focal plane tilt with the mirror tilt. Zemax ${ }^{\circledR}$, however, reports that the focal plane is aligned with the primary mirror.

Interestingly, seventh-order effects become important for the measurement of the spherical and fifth spherical, second coma, and second spherical aberrations. For each of these aberrations, the next order aberration intrinsic to the aligned telescope (seventh-order spherical, seventh-order second coma, and seventh-order second spherical) is of a similar magnitude or greater than the symmetric fifth-order aberration. Fortunately for telescope alignment, the corresponding seventh-order misalignment aberrations are smaller than the fifth-order misalignment aberrations.

\section{Wavefront Sensors: How Many, Where and to What Order?}

\subsection{General considerations}

In $₫ 2$ and $\$ 4$ we showed that telescope misalignments produce a finite and relatively small set of distinct aberration patterns, each of which is characterized by a two-vector. The degrees of freedom that produce these patterns are likewise finite and small in number.

Suppose that we have an $N$-mirror telescope with $n$ wavefront sensors distributed throughout the field each capable of measuring $m$ aberrations. We take the position and pointing of the telescope to be defined by one of the mirrors. There remain $N-1$ tilts and $N-1$ decenters, each of which is described by a two-vector, that can produce our misalignment aberration patterns. If the aberration patterns are linearly independent, one would need to measure and correct $2(N-1)$ misalignment patterns to keep the telescope aligned. 


\subsection{A naive approach to the 3-mirror telescope using trefoil}

For a 3-mirror telescope one would certainly measure the coma, astigmatism, and curvature of field patterns, since these produce the largest aberrations. The choice of a fourth pattern is much less obvious, but for the sake of the present exposition, we choose trefoil (despite its relatively small amplitude compared to several other fifth order aberrations).

Coma, astigmatism and trefoil each have two independent components on the pupil: one that varies as $\cos \phi, \cos 2 \phi$ and $\cos 3 \phi$ respectively and another that varies as $\sin \phi, \sin 2 \phi$ and $\sin 3 \phi$ respectively. Thus, if the Seidel patterns and effects of despace errors and mirror deformations are well-constrained, one wavefront sensor alone will suffice to measure the two-vectors for the misalignment aberration patterns associated with these.

By contrast, defocus is radially symmetric on the pupil, and each wavefront sensor produces only one number. Two wavefront sensors suffice to measure the curvature of field pattern, provided that they are not diametrically opposed.

The situation is complicated by despace (piston) errors which produce the symmetric aberration patterns characteristic of aligned but incompletely corrected telescopes. One would certainly want to measure and correct the first-order defocus that this produces, but this demands a third wavefront sensor to distinguish between simple (uniform) defocus and curvature of field.

One possible arrangement of these three wavefront sensors would be to space the wavefront sensors around the periphery of the field, where the effects of the misalignments are largest. An alternative would be to have one wavefront sensor at the center of the field and two at the periphery, but not co-linear.

As there are two degrees of freedom for despace errors, one must measure a second field-symmetric pattern in addition to defocus. The three candidate patterns are coma, astigmatism and spherical aberration. Only two wavefront sensors are needed to distinguish between the third-order misalignment coma and the symmetric coma pattern characteristic of a telescope with despace errors. The same holds true for astigmatism. The three wavefront sensors needed to distinguish uniform defocus and curvature of field therefore provide information about both despace errors. In addition, one would obtain three measurements of spherical aberration, which at third order is constant across the field.

Our naive scheme has one major strength and one major weakness. The strength is that the aberrations are low order on the pupil. Wavefront sensors work by subdividing the pupil

and making measurements on those elements. The more finely one subdivides the pupil, the more light is needed. Moreover, high-order aberrations are likely to be small, so there is 
little danger of our low order aberrations being corrupted by covariances with higher order aberrations. Finally, for the case of wavefront sensors based on out-of-focus images, image overlap is less of a problem if one can work with images that are more nearly in focus, which this method allows.

The substantial weakness in our scheme is the use of trefoil, which, as we have noted, would appear to produce a substantially smaller signal than some of the other fifth-order misalignment aberrations.

\section{3. alternatives to trefoil: second coma, astigmatism and spherical}

Tessieres' results, shown in Table 3, would suggest second coma as an alternative to trefoil. But as is evident if Figure 7, the point spread functions for second coma and ordinary (third order) coma are quite similar to each other. And the misalignment patterns, shown in Figures 2 and 8, are identical. So one would need to sample the pupil well in order to distinguish between the two.

Coma and second coma vary, respectively, as $\rho^{3} \cos \phi$ and $\rho^{5} \cos \phi$. We let

$$
C=\frac{\int_{r_{i}}^{r_{o}}\left(\rho^{5}\right)\left(\rho^{3}\right) \rho d \rho}{\left[\int_{r_{i}}^{r_{o}}\left(\rho^{5}\right)\left(\rho^{5}\right) \rho d \rho \int_{r_{i}}^{r_{o}}\left(\rho^{3}\right)\left(\rho^{3}\right) \rho d \rho\right]^{\frac{1}{2}}}
$$

be a measure of the correlation of the two wavefronts, where we have suppressed the azimuthal dependence and assumed that the center of the pupil is obstructed out to some fraction $r_{i}$ of its total radius. Taking $r_{i}=0.5$ and $r_{o}=1.0$ gives $C=0.981$, indicating a very strong correlation. By contrast $C$ is identically zero for any two of defocus, coma, astigmatism and trefoil.

As a consequence of this strong correlation, one needs very accurate wavefront sensing to keep the large third-order coma signal from corrupting the weaker second-coma signal.

The problem is exacerbated by the large central obscurations associated with wide-field telescopes. One must obtain at least two radial samples in the range $0.5<\rho<1.0$. For a Shack-Hartmann sensor, this would mean at least 8 lenslets across the diameter of the pupil. For out-of-focus wavefront sensing this would mean defocussing by at least 8 seeing disks.

The same argument holds for discriminating between ordinary (third order) astigmatism and second astigmatism, but with a slightly smaller correlation, $C=0.978$. And it holds again for discriminating between curvature of field and second spherical aberration, again with with $C=0.978$. 


\section{4. alternatives to trefoil: fifth-order coma and astigmatism}

The PSFs produced by the $\sigma$ and $\rho$ fifth-order misalignment coma are indistinguishable from those produced by third-order misalignment coma, but each of these produces substantially different field patterns. One can therefore hope to distinguish among them using additional wavefront sensors.

Since each of these patterns is characterized by a two-vector, and since each wavefront sensor gives only two numbers associated with the coma at that point, a bare minimum of three wavefront sensors are needed to distinguish among the three misalignment coma patterns.

Of the two alternative arrangements described in our naive approach, the one with all three wavefront sensors on the periphery fails to discriminate between the fifth-order $\rho$-coma pattern and the third-order misalignment coma pattern. By contrast the arrangement with one at the center and two non-colinear on the periphery does successfully discriminate among all three patterns.

One can still use only peripheral wavefront sensors as long as one was careful to include the contributions of misalignments to both the third-order coma pattern and fifth-order $\rho$ pattern. In solving for the misalignments, one might iterate, ignoring $\rho$-coma on the first iteration and then subtracting it off on subsequent iterations. But this would preclude counting $\rho$-coma as one of the $2(N-1)$ needed patterns.

The same arguments hold for the three misalignment astigmatism patterns as well. A strictly peripheral arrangement of wavefront cannot distinguish between the third-order misalignment astigmatism pattern and the fifth-order $\rho$ pattern.

\section{5. alternatives to fifth-order: pointing and distortion}

One could alternately choose to neglect fifth-order aberrations and instead use pointing or distortion to maintain alignment in a three mirror telescope.

Tilt (or pointing) is one of only two first-order aberrations. As its name implies, pointing uniformly shifts the location of every object in the field. It does not alter an image's point spread function and so it cannot be measured with a wavefront sensor unless the position of that wavefront sensor in the image plane is accurately known. In a paper on the Advanced Solar Telescope, Manuel \& Burge (2009) suggest that one might use pointing to constrain telescope alignment. This method would require accurate measurement of the position of the detector with respect to one of the mirrors. 
In the same way that spherical aberration might equally well have been called "second defocus" - the two have same dependence upon pupil azimuth - distortion might equally well have been called "second tilt." For a field with a pre-existing astrometric catalog, misalignment distortion can be measured directly from the science data or using two wavefront sensors whose positions are accurately determined. In the absence of a pre-existing catalog, one might also solve for the misalignment distortion patterns by comparing overlapping fields, as suggested by Sudol (2011). The distortion terms would then constrain four degrees of freedom of telescope misalignments.

\subsection{An improved approach to the 3-mirror telescope}

Both wavefront sensor arrangements adopted for our naive approach to the 3-mirror telescope suffice to keep it aligned, but not for the same reasons. For the arrangement with three peripheral wavefront sensors, either the $\sigma$-coma and $\sigma$-astigmatism is likely to give a stronger alignment signal than the trefoil pattern.

Alternatively, for the arrangement with one wavefront sensor at the center and two non-colinear sensors on the periphery, the $\rho$-coma and $\rho$-astigmatism can also be used. This would seem to give sufficient information to keep a 4-mirror telescope aligned, or a 3-mirror telescope with a corrector.

It should be noted, however, that if one measures all three misalignment coma patterns, one must add a fourth wavefront sensor if one also wants to measure the symmetric coma pattern due to despace errors. The same holds true for astigmatism.

Table 7 is a summary of misalignment and piston errors, and the number of wavefront sensors required to constrain them. The aberrations are divided into those measurable with very low order wavefront sensing, and those which require greater sampling of the pupil. Patterns that are degenerate in a single wavefront sensor and therefore require separate wavefront sensors to distinguish among them are grouped together. The P's and M's show the patterns one would measure with a minimal system of three wavefront sensors all at the same radius on the periphery: $\mathrm{P}$ denotes those patterns which can be used to constrain

piston; M misalignments. Six independent misalignment patterns are measured, more than sufficient for a three mirror telescope and barely sufficient for a four mirror telescope. 
Table 7. Wavefront sensing summary.

\begin{tabular}{lc}
\hline \hline \multicolumn{1}{c}{ VERY LOW ORDER WAVEFRONT SENSING } & \\
\hline $\begin{array}{l}\text { first-order symmetric defocus } \\
\text { third-order symmetric defocus (COF) } \\
\text { third-order misalignment defocus (COF) }\end{array}$ & $\mathrm{P}$ \\
third-order symmetric astigmatism & $\mathrm{M}^{\mathrm{a}}$ \\
third order misalignment astigmatism & $\mathrm{P}$ \\
fifth-order misalignment astigmatism- $\sigma$ & $\mathrm{M}$ \\
fifth-order misalignment astigmatism- $\rho$ & $\mathrm{M}$ \\
third-order symmetric coma & $\mathrm{b}$ \\
third order misalignment coma & $\mathrm{P}$ \\
fifth-order misalignment coma- $\sigma$ & $\mathrm{M}$ \\
fifth-order misalignment coma- $\rho$ & $\mathrm{M}$ \\
fifth-order misalignment trefoil & $\mathrm{b}$ \\
\hline \hline HIGHER ORDER WAVEFRONT SENSING & $\mathrm{M}$ \\
\hline third-order symmetric spherical & \\
fifth-order misalignment second-astigmatism & \\
fifth-order misalignment second-coma & \\
fifth-order misalignment spherical & \\
\hline
\end{tabular}

${ }^{a}$ Third-order misalignment defocus alone requires two wavefront sensors to fully constrain, even in the absence of first- or third-order symmetric defocus.

${ }^{\mathrm{b}}$ If only three wavefront sensors are used, the four astigmatism patterns and the four coma patterns are degenerate. But if the misalignment can be determined from the remaining patterns, one of these can be computed from the alignment solution. For rho-coma this degeneracy is broken by placing a wavefront sensor at the center of the field. However, the astigmatism degeneracy cannot be broken this way. 


\section{Complications}

\section{1. mirror deformation}

We have until now treated the mirrors of a telescope as rigid bodies. But under the influence of changing gravitational and thermal stresses, the mirror surfaces deform and influence the wavefront. Deformations of the mirrors can be expanded in terms of Zernike polynomials, but it is more efficient to expand them in terms of their elastic bending modes (Noethe 1991; Martin et al. 1998; Schechter et al. 2003).

If the stop is coincident with one of the mirrors, then the deformations of that mirror have the same effect on the wavefront at every point in the field. But if not, deformations of a mirror will project onto different parts of the pupil at different points in the field. Applying the same mirror deformation pattern to different mirrors will produce aberrated wavefronts that are offset with respect to each other. One must therefore sample the wavefront at relatively high density to ascertain which element is deformed.

Among the fifth-order aberrations, second-coma, second-astigmatism and fifth-order spherical likewise require good sampling of the pupil to distinguish their misalignment patterns from those of third order aberrations. The accuracy with which one might determine these fifth-order aberrations is diminished by the need to use the same information to determine the mirror deformations.

This would argue for using two kinds of wavefront sensors: low-order sensors, using only coma, astigmatism and defocus to correct the rigid body motions, and high-order sensors to correct for mirror deformations. These latter sensors might be run at lower cadence than the former, except perhaps immediately following a large change in telescope pointing.

In such a scheme one might still measure fifth-order misalignment coma and fifth-order misalignment astigmatism with the low-order wavefront sensors, provided that there are a sufficient number of such sensors. If the telescope is then properly aligned the secondcoma and second-astigmatism misalignment patterns will be zeroed out on the high-order wavefront sensors, giving a cleaner measurement of the mirror deformations in the high-order sensors.

\section{2. central obscuration}

Many wide field telescope designs have a large central obscuration. As mentioned in $\$ 5$, this would make it more difficult to distinguish between second coma and second astigmatism 
on the one hand and ordinary coma and astigmatism on the other, which in third-order have the same field pattern. The smaller range of pupil radii would increase correlated errors.

\section{3. focal plane misalignments}

We have until this point avoided the question of how one knows the position of one's wavefront sensors or one's field with respect to one or another of a telescope's mirrors. As noted in the discussion of pointing in the previous subsection, the position and tilt of the focal plane or wavefront sensors might be determined mechanically or interferometrically. But if not, and if one wishes to correct for focal plane misalignments, one must measure two additional aberration patterns, one for the tilt of the focal plane, and one for the decenter.

A tilted detector produces a field pattern identical to misalignment curvature of field. However, if that pattern is used to correct the detector tilt, it cannot also be used to keep the mirrors aligned.

A decentered focal plane creates no aberrations of its own, but as is discussed in $§ \$ 2.7$, it can cause Seidel or fifth-order aligned aberrations to masquerade as misalignment aberrations. Measuring telescope aberrations relative to an incorrect field center will produce spurious misalignment aberrations even if the telescope is otherwise aligned.

One can choose to fit for this focal plane decenter by measuring an additional field pattern. If it is not feasible to measure an additional field pattern and one assumes that the center of the detector is the center of pointing, he can still completely null the measured misalignment coma, astigmatism and curvature of field by tilting and decentering one of the mirrors and tilting the focal plane. Compensating for the detector decenter with mirror misalignments can put the telescope into a state of 'benign misalignment', where only the smaller aberrations patterns: misalignment distortion, the fifth-order misalignment aberrations, and the aberrations which vary as the square of misalignments are present.

\section{4. transmitting correctors}

In his treatment of the LSST, Tessieres (2003) studies the effects of tilts and decenters on a focal plane assembly consisting of a multi-element corrector and the focal plane array. Two additional patterns are needed to keep this assembly aligned. Interestingly, the fifthorder aberration patterns produced by these tilts and decenters are larger, compared to the third-order aberration patterns, than for the secondary and tertiary mirrors. 


\section{Using and not using misalignment aberration patterns}

At least three of the generic misalignment aberration patterns described in the previous sections are currently used to align wide-field telescopes, and there may soon be reason to use more of them. Ignoring for the present, the question of whether or not to use distortion 11 we imagine here an $N$-mirror telescope with $n$ wavefront sensors distributed throughout the field each capable of measuring $m$ aberrations. One must determine $N-1$ tilts and $N-1$ decenters, each of which is described by a two-vector.

\section{1. independent analysis of the wavefront sensors}

The most straightforward and transparent approach is to analyze each wavefront sensor separately, determining the coefficients of the aberrations at each of $m$ points in the field. One then fits the these coefficients to a linear combinations of the misalignment aberration patterns described in the previous section and finally fits the amplitudes of the misalignment patterns (assuming one has measured more patterns than one actually needs) 12

A complication of this approach is that at each step the quantities for which one is fitting may be correlated with each other. Aberration coefficients will be correlated, pattern amplitudes will be correlated and tilts and decenters will be correlated. Under such circumstances one must be careful to fit for all correlated quantities; otherwise one runs the risk of introducing systematic errors in the quantities for which one does fit.

\section{2. $\quad$ simultaneous fit of all wavefront sensors for pattern amplitudes}

Instead of measuring the aberrations at each point in the field, one might fit the data for all $n$ wavefront sensors simultaneously to determine the amplitudes of the misalignment aberration patterns. This has the advantage of eliminating the problem of correlated aberrations at each point, but unmodelled aberrations may then lead to systematic errors.

\footnotetext{
${ }^{11}$ We take the view what can be measured can hurt you.

${ }^{12}$ It would not be surprising if two or more patterns were produced by the same, or nearly the same combination of tilts and decenters.
} 


\section{3. $\quad$ simultaneous fit of all wavefront sensors for tilts and decenters}

One might also fit directly for the tilts and decenters, short-circuiting the misalignment aberration patterns except for using them to turn tilts and decenters into predicted wavefronts that are then compared with observed wavefronts. This reduces the number of parameters for which one fits and implicitly accounts for the correlations of aberration pattern amplitudes. Rather than fitting for poorly determined amplitudes of fifth-order aberration patterns, all of the fifth-order dependence is attributed to a smaller number of tilts and decenters, which are strongly constrained by the third-order aberration patterns.

\section{4. forget about misalignment patterns}

Finally one might dispense entirely with the decomposition of the wavefront into specific aberrations and instead use ray tracing to determine how it varies at each measured point. While this obviates the need for misalignment patterns, it sacrifices all understanding of why one might need $n$ wavefront sensors with enough resolution to measure $m$ aberrations.

\section{Summary}

We have derived and illustrated the generic third-order aberration patterns that arise when the axial symmetry of a telescope is broken by small misalignments of optical elements. There are five patterns: one each for coma, astigmatism and curvature of field and two for distortion. Each of these misalignment patterns is characterized by an associated twodimensional vector. These two-vectors are in turn linear combinations of the tilt and decenter vectors of the individual optical elements.

For an $N$-mirror telescope, $2(N-1)$ patterns must be measured to keep the telescope aligned. For $N=3$, as in a three mirror anastigmat, there is a two-dimensional "subspace of benign misalignment" over which the misalignment patterns for third-order coma, astigmatism and curvature of field are identically zero. If pre-existing astrometry is available, one or both of the distortion patterns may be used to keep the telescope aligned. Alternatively, one might measure one of the fifth-order misalignment patterns.

We have illustrated the generic fifth-order misalignment patterns that arise from small misalignments. These are relatively insensitive to misalignments and may be of little use in telescope alignment. One would appear to be driven back to using distortion, or alternatively, pointing. 
Acknowledgements: We gratefully acknowledge helpful and thought provoking conversations and communications with Michael Jarvis, Don Phillion, Lothar Noethe, Stephen Shectman and Tony Tyson. We also thank the referee, Brian McLeod, for his extensive comments. This work was supported by the National Science Foundation through a Graduate Research Fellowship to Rebecca Sobel Levinson

\section{REFERENCES}

Gitton, P., \& Noethe, L. 1998, The Messenger, 92, 15

Hopkins, H. H. 1950, Clarendon Press, Oxford.

Hvisc, A. M., \& Burge, J. H. 2008, Proc. SPIE, 7018,

Lee, H., Dalton, G., Tosh, I.A.J,, \& Kim, S.-W. 2008, Opt. Exp., 16, 10992.

Ma, Z., Bernstein, G., Weinstein, A., \& Sholl, M. 2008, PASP, 120, 1307

Manuel, A. 2009, Ph.D. dissertation, University of Arizona, Tucson, Arizona.

Manuel, A. M., \& Burge, J. H. 2009, Proc. SPIE, 7433

Maréchal, A., 1950, Rev. Opt., 29, 1

Martin, H. M., et al. 1998, Proc. SPIE, 3352, 412

McLeod, B. A. 1996, PASP, 108, 217

Noethe, L. 1991, J. Mod. Opt., Vol. 38, No. 6, p. 1043 - 1066, 38, 1043

Noethe, L., \& Guisard, S. 2000, Proc. SPIE, 4003, 382

Palunas, P., et al. 2010, Proc. SPIE, 0, 0000

Phillion, D. W., Olivier, S. S., Baker, K., Seppala, L., \& Hvisc, S. 2006, Proc. SPIE, 6272

Pier, J. R., Munn, J. A., Hindsley, R. B., Hennessy, G. S., Kent, S. M., Lupton, R. H., \& Ivezić, Ž. 2003, AJ, 125, 1559

Romano, A., et al. 2010, A\&A, 514, A88

Schechter, P. L., et al. 2003, Proc. SPIE, 4837, 619

Schroeder, D. J. 1987, San Diego, CA, Academic Press, Inc, 363 p. 
Seppala, L. G. 2002, Proc. SPIE, 4836, 111

Shack, R. V., \& Thompson, K. 1980, Proc. SPIE, 251, 146

Sudol, J. 2011, private communications

Tessieres, R. 2003, M.S. thesis, University of Arizona, Tucson, Arizona.

Thompson, K. 1980, Ph.D. dissertation, University of Arizona, Tucson, Arizona.

Thompson, K. 2005, J. Opt. Soc. Am. A, 22, 1389

Thompson, K., et al. 2009, J. Opt. Soc. Am. A, 26, 1503

Wilson, R. N. 1996, Springer-Verlag Berlin Heidelberg New York, 543 p.

Wilson, R. N., \& Delabre, B. 1997, PASP, 109, 53

Chandra X-ray Center 2009, The Chandra Proposer's Observatory Guide, Version 12. Retrieved from http://cxc.harvard.edu/proposer/POG/

\section{A. Coma}

Schroeder (1987), calculates the the coma pattern cause by tilting and decentering the secondary of a two mirror telescope by amounts $\alpha$ and $\ell$.

$$
\begin{aligned}
G & =B_{2} \rho^{3} \sin \phi \\
B_{2} & =B_{2}(\text { cen })+\frac{1}{R_{2}^{2}}\left[\frac{l}{R_{2}}\left[K_{2}-\left(\frac{m_{2}+1}{m_{2}-1}\right)\right]-\alpha\left(\frac{m_{2}+1}{m_{2}-1}\right)\right] \\
B_{2}(\text { cen }) & =\frac{\theta}{R_{1}^{2}}-\frac{W \theta}{R_{2}^{2}}\left[\left(\frac{m_{2}+1}{m_{2}-1}\right)\left(\frac{1}{W}-\frac{1}{R_{2}}\right)+\frac{K_{2}}{R_{2}}\right]
\end{aligned}
$$

where $\rho$ and $\phi$ are the radial and angular coordinates on the pupil and $\theta$ is the radial coordinate of the image in the field. The quantities $R_{i}, K_{i}$ and $m_{i}$ are all mirror properties: the radius of curvature, conic constant, and magnification of a mirror, where the subscript denotes which mirror is being addressed. The index of refraction has been set equal to 1 
for the primary mirror and -1 for the secondary mirror. Finally, $W$ is the distance from the primary mirror to the secondary.

$B_{2}($ cen $)$ is the coma of an aligned two mirror telescope. For the sake of simplicity the tilt and decenter of the secondary from the primary, and the object displacement from the optical axis have been taken lie along the $y$ axis in Schroeder's analysis.

Schroeder's equations can be generalized for an object displaced from the optical axis in an arbitrary direction. The chief ray for the object is given by $\vec{\sigma}$ with radial and angular components $\sigma$ and $\theta$. The equations can be further generalized to allow decentering and tilting of the secondary mirror in arbitrary directions $\vec{\ell}$ and $\vec{\alpha}$ with radial components $\ell$ and $\alpha$ and angular components $\phi_{\ell}$ and $\phi_{\alpha}$. The equations become:

$$
G=B_{2 x} \rho^{3} \cos \phi+B_{2 y} \rho^{3} \sin \phi
$$

where the field dependences are given by

$$
\begin{aligned}
B_{2 x} & =G_{\text {Seidel }}^{\text {coma }} \sigma \cos \theta+G_{\text {decenter }}^{\text {coma }} \cos \phi_{\ell}+G_{\text {tilt }}^{\text {coma }} \alpha \cos \phi_{\alpha} \\
B_{2 y} & =G_{\text {Seidel }}^{\text {coma }} \sigma \sin \theta+G_{\text {decenter }}^{\text {coma }} \ell \sin \phi_{\ell}+G_{\text {tilt }}^{\text {coma }} \alpha \sin \phi_{\alpha} \\
G_{\text {Seidel }}^{\text {coma }} & =\frac{1}{R_{1}^{2}}-\frac{W}{R_{2}^{2}}\left[\left(\frac{m_{2}+1}{m_{2}-1}\right)\left(\frac{1}{W}-\frac{1}{R_{2}}\right)+\frac{K_{2}}{R_{2}}\right] \\
G_{\text {decenter }}^{\text {coma }} & =\frac{1}{R_{2}^{3}}\left[K_{2}-\left(\frac{m+1}{m-1}\right)\right] \\
G_{\text {tilt }}^{\text {coma }} & =-\frac{1}{R_{2}^{3}}\left(\frac{m+1}{m-1}\right)
\end{aligned}
$$

Consolidating the above equations yields

$$
G^{\text {coma }}=G_{\text {decenter }}^{c o m a} \sigma \rho^{3} \cos (\phi-\theta)+G_{\text {decenter }}^{\text {coma }} \ell \rho^{3} \cos \left(\phi-\phi_{\ell}\right)+G_{\text {tilt }}^{\text {coma }} \alpha \rho^{3} \cos \left(\phi-\phi_{\alpha}\right)
$$

which is the form for the coma aberration given in $\$ 2$

\section{B. Astigmatism}

McLeod (1996) follows the notation of Schroeder and calculates the astigmatism pattern for a two mirror telescope for which the secondary mirror has been aligned (decentered) to 
null the field constant coma pattern. McLeod gives the form of the remaining astigmatism patterns as

$$
\begin{gathered}
W=Z_{4} \rho^{2} \cos 2 \phi+Z_{5} \rho^{2} \sin 2 \phi \\
Z_{4}=B_{0}\left(\theta_{x}^{2}-\theta_{y}^{2}\right)+B_{1}\left(\theta_{x} \alpha_{x}-\theta_{y} \alpha_{y}\right)+B_{2}\left(\alpha_{x}^{2}-\alpha_{y}^{2}\right) \\
Z_{5}=2 B_{0}\left(\theta_{x} \theta_{y}\right)+B_{1}\left(\theta_{x} \alpha_{y}+\theta_{y} \alpha_{x}\right)+2 B_{2}\left(\alpha_{x} \alpha_{y}\right) \\
B_{0}=A_{0}^{p} r_{p}^{2}-A_{0}^{s} r_{s}^{2} \\
B_{1}=-r_{s}^{2}\left[2 A_{0}^{s}+\left(W+d_{n}\right) A_{1}^{s}\right] \\
B_{2}=-r_{s}^{2}\left[A_{0}^{s}+\left(W+d_{n}\right) A_{1}^{s}+\left(W+d_{n}\right)^{2} A_{2}^{s}\right] \\
A_{0}=\frac{W^{2}}{2 R}\left[\frac{K}{R^{2}}+\left(\frac{1}{W}-\frac{1}{R}\right)^{2}\right] \\
A_{1}=\frac{W}{R^{2}}\left[\frac{1}{W}-\frac{K+1}{R}\right] \\
A_{2}=\frac{K+1}{2 R^{3}}
\end{gathered}
$$

where $\rho$ and $\phi$ are the (normalized) radial and angular coordinates on the pupil and $\theta_{x}$ and $\theta_{y}$ are the cartesian coordinates of the image in the field. The quantities $R_{i}, K_{i}$ and $m_{i}$ are again all mirror properties: the radius of curvature, conic constant, and magnification of a mirror, where the subscript denotes which mirror is being addressed, the primary $p$, or the secondary $s . W$ is the distance from the primary mirror to the secondary, and $\alpha_{x}$ and $\alpha_{y}$ are the tilts of the secondary mirror with respect to the primary mirror. The indices of refraction preceding the primary and secondary mirrors have been set to 1 and -1 respectively.

McLeod additionally uses two constants that are not present in Schroeder's analysis of the coma pattern: $r_{i}$, which is the marginal ray height at the optic, and $d_{n}$, which denotes the position of the coma free point. As McLeod nulled the misalignment coma prior to analyzing the astigmatism, the decenter of the system $\vec{\ell}$ is given by $\vec{\ell}=d_{n} \vec{\alpha}$. McLeod chose to express the astigmatism only in terms of the tilt of the secondary mirror, though he could have equivalently expressed the astigmatism only in terms of the decenter or as a combination of the two terms. For greater transparency of the field patterns caused by both decenters and tilts, we here decouple the decenter and tilt terms in McLeod's equations, removing the variable $d_{n}$. We also de-normalize the pupil coordinates and remove the terms which vary as the square of the misalignment. 


$$
\begin{gathered}
W=Z_{4} \rho^{2} \cos 2 \phi+Z_{5} \rho^{2} \sin 2 \phi \\
Z_{4}=B_{0}\left(\theta_{x}^{2}-\theta_{y}^{2}\right)+B_{1 \text { decenter }}\left(\theta_{x} \ell_{x}-\theta_{y} \ell_{y}\right)+B_{1 t i l t}\left(\theta_{x} \alpha_{x}-\theta_{y} \alpha_{y}\right) \\
Z_{5}=2 B_{0}\left(\theta_{x} \theta_{y}\right)+B_{1 \text { decenter }}\left(\theta_{x} \ell_{y}+\theta_{y} \ell_{x}\right)+B_{1 \text { tilt }}\left(\theta_{x} \alpha_{y}+\theta_{y} \alpha_{x}\right) \\
B_{0}=A_{0}^{p} r_{p}^{2}-A_{0}^{s} r_{s}^{2} \\
B_{1 \text { decenter }}=2 A_{0}^{s}+W A_{1}^{s} \\
B_{1 \text { tilt }}=A_{1}^{s} \\
A_{0}=\frac{W^{2}}{2 R}\left[\frac{K}{R^{2}}+\left(\frac{1}{W}-\frac{1}{R}\right)^{2}\right] \\
A_{1}=\frac{W}{R^{2}}\left[\frac{1}{W}-\frac{K+1}{R}\right]
\end{gathered}
$$

By converting the field variables to polar coordinates $\sigma$ and $\phi_{\sigma}$ and similarly converting the misalignment variables to polar form $\ell, \phi_{\ell}, \alpha, \phi_{\alpha}$, the expression for the wavefront delay yields the form given in $₫ 2$,

$G^{a s t i g}=G_{\text {Seidel }}^{\text {astig }} \sigma^{2} \rho^{2} \cos 2(\phi-\theta)+G_{\text {decenter }}^{\text {astig }} \sigma \ell \rho^{2} \cos \left(2 \phi-\theta-\phi_{\ell}\right)+G_{\text {tilt }}^{\text {astig }} \sigma \alpha \rho^{2} \cos \left(2 \phi-\theta-\phi_{\alpha}\right)$

where $B_{0}, B_{1 \text { decenter }}$ and $B_{1 \text { tilt }}$ are equal to $G_{\text {Seidel }}^{\text {astig }}, G_{\text {decenter }}^{\text {astig }}$ and $G_{\text {tilt }}^{\text {astig }} .13$

\section{Curvature of Field}

The misalignment patterns for curvature of field are less well-explored in the literature. Thompson (2005) presents forms for the patterns, and we re-derive them here from the despaces and misalignments of a single mirror from the pupil.

As stated in 92.6 , the contribution of a single mirror to the wavefront delay of the ray which strikes the mirror at position $\vec{\varpi}$ and makes an angle $\vec{\psi}$ with the axis of the mirror is given by:

\footnotetext{
${ }^{13}$ The form of the astigmatism field pattern holds even for telescopes which have not been aligned to null the misalignment coma pattern. The expanded coefficients for the wavefront delay of a randomly misaligned telescope can be found in Table 2
} 


$$
\begin{aligned}
G^{3 r d} & =W_{040}\left(\frac{\vec{\varpi}}{R} \cdot \frac{\vec{\varpi}}{R}\right)\left(\frac{\vec{\varpi}}{R} \cdot \frac{\vec{\varpi}}{R}\right)+W_{131}\left(\vec{\psi} \cdot \frac{\vec{\varpi}}{R}\right)\left(\frac{\vec{\varpi}}{R} \cdot \frac{\vec{\varpi}}{R}\right) \\
& +W_{222}\left(\vec{\psi} \cdot \frac{\vec{\varpi}}{R}\right)\left(\vec{\psi} \cdot \frac{\vec{\varpi}}{R}\right)+W_{220}(\vec{\psi} \cdot \vec{\psi})\left(\frac{\vec{\varpi}}{R} \cdot \frac{\vec{\varpi}}{R}\right) \\
& +W_{311}(\vec{\psi} \cdot \vec{\psi})\left(\vec{\psi} \cdot \frac{\vec{\varpi}}{R}\right)
\end{aligned}
$$

where $W_{040}$ is the spherical aberration coefficient, $W_{131}$ is the coma coefficient, $W_{222}$ is the astigmatism coefficient, $W_{220}$ is the curvature of field coefficient, and $W_{311}$ is the distortion coefficient. These aberration coefficients depend only on the curvature of the mirror, $R$, the conic constant of the mirror, $K$, the magnification of the mirror, $m$, the index of refraction of the air preceding the mirror, $n$ and the position of the object for the mirror, $s$.

The transformations from pupil coordinates $\vec{\rho}$ and $\vec{\sigma}$ to mirror coordinates for a mirror despaced by an amount $W$ and decentered and tilted by $\vec{l}$ and $\vec{\alpha}$ are

$$
\begin{aligned}
\vec{\psi} & =\left(1-\frac{W}{s}\right) \vec{\sigma}-\left(\vec{\alpha}+\frac{\vec{l}}{s}\right) \\
\vec{\varpi} & =(\vec{\rho}-W \vec{\psi})-\vec{l} .
\end{aligned}
$$

Expanding the wavefront delay caused by a single mirror in terms of the pupil coordinates and keeping only those terms which vary as $\rho^{2}$ on the pupil 14 and vary linearly with the misalignments or less, we find an expression for the curvature of field wavefront delay added by a single offset mirror.

$$
\begin{aligned}
G_{i}^{C O F}=[ & \left.\frac{1}{2}\left(\frac{W}{s}-1\right)^{2} W_{220}+\frac{W}{R}\left(\frac{W}{s}-1\right) W_{131}+2 \frac{W^{2}}{R^{2}} W_{040}\right]\left(\frac{\vec{\rho}}{R} \cdot \frac{\vec{\rho}}{R}\right)(\vec{\sigma} \cdot \vec{\sigma}) \\
+ & {\left[2\left(\frac{R}{s}\right)\left(\frac{W}{s}+1\right) W_{220}+\left(\frac{2 W}{s}+1\right) W_{131}+4 \frac{W}{R} W_{040}\right]\left(\frac{\vec{\rho}}{R} \cdot \frac{\vec{\rho}}{R}\right)\left(\vec{\sigma} \cdot \frac{\vec{l}}{R}\right) } \\
& +\left[2\left(\frac{W}{s}+1\right) W_{220}+\frac{W}{R} W_{131}\right]\left(\frac{\vec{\rho}}{R} \cdot \frac{\vec{\rho}}{R}\right)(\vec{\sigma} \cdot \vec{\alpha})
\end{aligned}
$$

\footnotetext{
${ }^{14}$ The astigmatic component of the wavefront additionally has terms which vary as $\rho^{2}$ on the pupil. We exclude these astigmatic terms in the analysis of COF
} 
For a two mirror telescope, the primary and the secondary both contribute to the wavefront delay. As the primary is neither despaced nor misaligned from the pupil, the form of its contribution to the wavefront delay is simplified; notably, the primary mirror only contributes to the Seidel aberration. The secondary mirror is despaced and possibly misaligned from its pupil and therefore contributes to the decenter and tilt terms as well as the Seidel pattern. Combining the effects of the primary and secondary mirror yields:

$$
\begin{gathered}
G^{C O F}=G_{\text {Seidel }}^{C O F}(\vec{\sigma} \cdot \vec{\sigma})(\vec{\rho} \cdot \vec{\rho})+G_{\text {decenter }}^{C O F}(\vec{\sigma} \cdot \vec{\ell})(\vec{\rho} \cdot \vec{\rho})+G_{\text {tilt }}^{C O F}(\vec{\sigma} \cdot \vec{\alpha})(\vec{\rho} \cdot \vec{\rho}) \\
G_{\text {Seidel }}^{C O F}=R_{1}^{2} W_{220_{1}}+R_{2}^{2}\left[\frac{1}{2}\left(\frac{W}{s_{2}}-1\right)^{2} W_{220_{2}}+\frac{W}{R_{2}}\left(\frac{W}{s_{2}}-1\right) W_{131_{2}}+2 \frac{W^{2}}{R_{2}^{2}} W_{040_{2}}\right] \\
G_{\text {decenter }}^{C O F}=-R_{2}^{3}\left[2\left(\frac{R_{2}}{s_{2}}\right)\left(\frac{W}{s_{2}}+1\right) W_{220_{2}}+\left(\frac{2 W}{s_{2}}+1\right) W_{131_{2}}+4 \frac{W}{R_{2}} W_{040_{2}}\right] \\
G_{\text {tilt }}^{C O F}=-R_{2}^{2}\left[2\left(\frac{W}{s_{2}}+1\right) W_{20_{2}}+\frac{W}{R_{2}} W_{131_{2}}\right]
\end{gathered}
$$

which is the form for the COF wavefront delay that appears in $\$ 2$.

\section{Distortion}

The distortion field patterns can additionally be derived from the wavefront delay caused by a single optic. Again expanding equation (C1) in terms of pupil coordinates, but now retaining only those terms which vary as $\rho$ on the pupil and vary linearly with the misalignments or less yields the expression for the distortion contribution of a single mirror which is despaced by $W$, decentered by $\vec{\ell}$ and tilted by $\vec{\alpha} .15$

\footnotetext{
${ }^{15}$ As noted in 92 , we have omitted the $W_{311}$ coefficient as it is equal to zero.
} 


$$
\begin{aligned}
G_{i}^{\text {distortion }}=- & {\left[2 \frac{W}{R}\left(\frac{W}{s}-1\right)^{2}\left(W_{220}+W_{222}\right)+3 \frac{W^{2}}{R^{2}}\left(\frac{W}{s}-1\right) W_{131}+4 \frac{W^{3}}{R^{3}} W_{040}\right]\left(\frac{\vec{\rho}}{R} \cdot \vec{\sigma}\right)(\vec{\sigma} \cdot \vec{\sigma}) } \\
& -\left[4\left(\frac{W}{s}\right)\left(\frac{W}{s}-1\right) W_{220}+2\left(\frac{W}{s}-1\right)\left(\frac{2 W}{s}-1\right) W_{222}\right. \\
& -\left[2 \frac{W}{R}\left(\frac{W}{s}-1\right)\left(2 W_{220}+W_{222}\right)+2 \frac{W^{2}}{R^{2}} W_{131}\right]\left(\frac{3 W}{2 s}-\vec{\rho}\right)(\vec{\sigma} \cdot \vec{\alpha}) \\
& -\left[2\left(\frac{W}{s}-1\right)^{2} W_{220}+2\left(\frac{W}{s}\right)\left(\frac{W}{s}-1\right) W_{222}\right. \\
& -\left[2 \frac{W}{R}\left(\frac{W}{s}-1\right) W_{222}+\frac{W^{2}}{R^{2}} W_{131}\right]\left(\frac{\vec{\rho}}{R} \cdot \vec{\alpha}\right)(\vec{\sigma} \cdot \vec{\sigma})
\end{aligned}
$$

For a two mirror telescope, only the secondary contributes to the distortion wavefront delay, as the primary is by construct not despaced from its entrance pupil. Grouping the coefficients in the above equation into terms of field dependences yields the form for distortion in $§ 2$ :

$$
\begin{aligned}
G^{\text {distortion }} & =G_{\text {Seidel }}^{\text {distion }} \sigma^{3} \rho \cos (\phi-\theta) \\
& +G_{\text {decenter }, \sigma}^{\text {distortion }} \sigma^{2} \ell \cos \left(\theta-\phi_{\ell}\right) \rho \cos (\phi-\theta)+G_{\text {decenter }, \rho}^{\text {distortion }} \sigma^{2} \ell \rho \cos \left(\phi-\phi_{\ell}\right) \\
& +G_{\text {tilt }, \sigma}^{\text {distortion }} \sigma^{2} \alpha \cos \left(\theta-\phi_{\alpha}\right) \rho \cos (\phi-\theta)+G_{\text {tilt }, \rho}^{\text {distion }} \sigma^{2} \alpha \rho \cos \left(\phi-\phi_{\alpha}\right)
\end{aligned}
$$

The coefficients are given by: 


$$
\begin{aligned}
G_{\text {Seidel }}^{\text {distortion }}=\frac{1}{R}\left[2 \frac{W}{R}\left(\frac{W}{s}-1\right)^{2}\left(W_{220}+W_{222}\right)+3 \frac{W^{2}}{R^{2}}\left(\frac{W}{s}-1\right) W_{131}+4 \frac{W^{3}}{R^{3}} W_{040}\right] \\
G_{\text {decenter }, \sigma}^{\text {distortion }}=-\frac{1}{R^{2}}\left[4\left(\frac{W}{s}\right)\left(\frac{W}{s}-1\right) W_{220}+2\left(\frac{W}{s}-1\right)\left(\frac{2 W}{s}-1\right) W_{222}\right. \\
\left.\quad+4 \frac{W}{R}\left(\frac{3 W}{2 s}-1\right) W_{131}+8 \frac{W^{2}}{R^{2}} W_{040}\right] \\
G_{\text {decenter }, \rho}^{\text {distortion }}=-\frac{1}{R^{2}}\left[2\left(\frac{W}{s}-1\right)^{2} W_{220}+2\left(\frac{W}{s}\right)\left(\frac{W}{s}-1\right) W_{222}\right. \\
\left.\quad+2 \frac{W}{R}\left(\frac{3 W}{2 s}-1\right) W_{131}+4 \frac{W^{2}}{R^{2}} W_{040}\right] \\
G_{\text {tilt }, \sigma}^{\text {distion }}=-\frac{1}{R}\left[2 \frac{W}{R}\left(\frac{W}{s}-1\right)\left(2 W_{220}+W_{222}\right)+2 \frac{W^{2}}{R^{2}} W_{131}\right] \\
G_{\text {tilt }, \rho}^{\text {distion }}=-\frac{1}{R}\left[2 \frac{W}{R}\left(\frac{W}{s}-1\right) W_{222}+\frac{W^{2}}{R^{2}} W_{131}\right]
\end{aligned}
$$

where all mirror properties and object distances refer to the secondary mirror, and the chief ray angle is the chief ray angle on the primary mirror. 